\title{
Genome-wide identification of the Liriodendron chinense WRKY gene family and its diverse roles in response to multiple abiotic stress
}

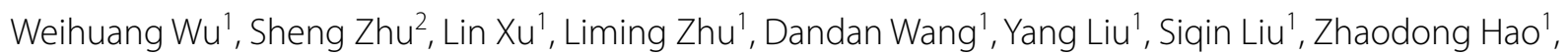
Ye Lu' ${ }^{1}$, Liming Yang ${ }^{2}$, Jisen Shi ${ }^{1}$ and Jinhui Chen ${ }^{1^{*}}$

\begin{abstract}
Background: Liriodendron chinense (LChi) is a tree species within the Magnoliaceae family and is considered a basal angiosperm. The too low or high temperature or soil drought will restrict its growth as the adverse environmental conditions, thus improving L. chinense abiotic tolerance was the key issues to study. WRKYs are a major family of plant transcription factors known to often be involved in biotic and abiotic stress responses. So far, it is still largely unknown if and how the LChiWRKY gene family is tied to regulating $L$. chinense stress responses. Therefore, studying the involvement of the WRKY gene family in abiotic stress regulation in L. chinense could be very informative in showing how this tree deals with such stressful conditions.
\end{abstract}

Results: In this research, we performed a genome-wide analysis of the Liriodendron chinense (LChi) WRKY gene family, studying their classification relationships, gene structure, chromosomal locations, gene duplication, cis-element, and response to abiotic stress. The 44 members of the LchiWRKY gene family contain a significant amount of sequence diversity, with their lengths ranging from 525 bp to 40,981 bp. Using classification analysis, we divided the 44 LchiWRKY genes into three phylogenetic groups (I, II, II), with group II then being further divided into five subgroups (Ila, IIb, IIc, IId, Ile). Comparative phylogenetic analysis including the WRKY families from 17 plant species suggested that LchiWRKYs are closely related to the Magnolia Cinnamomum kanehirae WRKY family, and has fewer family members than higher plants. We found the LChiWRKYS to be evenly distributed across 15 chromosomes, with their duplication events suggesting that tandem duplication may have played a major role in LchiWRKY gene expansion model. A Ka/Ks analysis indicated that they mainly underwent purifying selection and distributed in the group Ild. Motif analysis showed that LchiWRKYs contained 20 motifs, and different phylogenetic groups contained conserved motif. Gene ontology (GO) analysis showed that LChiWRKYS were mainly enriched in two categories, i.e., biological process and molecular function. Two group IIc members (LchiWRKY10 and LchiWRKY37) contain unique WRKY element sequence variants (WRKYGKK and WRKYGKS). Gene structure analysis showed that most LchiWRKYs possess 3 exons and two different types of introns: the R- and V-type which are both contained within the WRKY domain (WD). Additional promoter cis-element analysis indicated that 12 cis-elements that play different functions in environmental adaptability occur

\footnotetext{
*Correspondence: chenjh@njfu.edu.cn

${ }^{1}$ Key Laboratory of Forest Genetics and Biotechnology, Ministry

of Education of China, Co-Innovation Center for the Sustainable Forestry

in Southern China, Nanjing Forestry University, Nanjing, China

Full list of author information is available at the end of the article
}

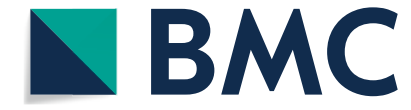

(C) The Author(s) 2022. Open Access This article is licensed under a Creative Commons Attribution 4.0 International License, which permits use, sharing, adaptation, distribution and reproduction in any medium or format, as long as you give appropriate credit to the original author(s) and the source, provide a link to the Creative Commons licence, and indicate if changes were made. The images or other third party material in this article are included in the article's Creative Commons licence, unless indicated otherwise in a credit line to the material. If material is not included in the article's Creative Commons licence and your intended use is not permitted by statutory regulation or exceeds the permitted use, you will need to obtain permission directly from the copyright holder. To view a copy of this licence, visit http://creativecommons.org/licenses/by/4.0/. The Creative Commons Public Domain Dedication waiver (http://creativeco mmons.org/publicdomain/zero/1.0/) applies to the data made available in this article, unless otherwise stated in a credit line to the data. 
across all LChiWRKY groups. Heat, cold, and drought stress mainly induced the expression of group II and I LChiWRKYS, some of which had undergone gene duplication during evolution, and more than half of which had three exons. LChiWRKY33 mainly responded to cold stress and LChiWRKY25 mainly responded to heat stress, and LchiWRKY18 mainly responded to drought stress, which was almost 4-fold highly expressed, while 5 LChiWRKYs (LChiWRKY5, LChiWRKY23, LChiWRKY14, LChiWRKY27, and LChiWRKY36) responded equally three stresses with more than 6-fold expression. Subcellular localization analysis showed that all LChiWRKYs were localized in the nucleus, and subcellular localization experiments of LChiWRKY18 and 36 also showed that these two transcription factors were expressed in the nucleus.

Conclusions: This study shows that in Liriodendron chinense, several WRKY genes like LChiWRKY33, LchiWRKY25, and LChiWRKY18, respond to cold or heat or drought stress, suggesting that they may indeed play a role in regulating the tree's response to such conditions. This information will prove a pivotal role in directing further studies on the function of the LchiWRKY gene family in abiotic stress response and provides a theoretical basis for popularizing afforestation in different regions of China.

Keywords: Liriodendron chinense, Genome-wide, WRKY gene family, Transcription factor, Abiotic stress

\section{Background}

Transcription factors (TFs) are important proteins that bind to specific DNA motifs to regulate gene expression and play crucial roles in plant growth, development, metabolism, and stress response [1,2]. WRKY transcription factors were first identified in plants and form one of their largest gene families [3]. After the first WRKY gene was cloned in sweet potato, the $W R K Y$ gene family function has been studied in several model plants, such as Arabidopsis thaliana (72), Oryza sativa (76), Amborella trichopoda (31), and Solanum lycopersicum (81) [4, 5]. The main defining feature of WRKY proteins is their WRKY domain, which contains a conserved amino acid sequence WRKYGQK at the $\mathrm{N}$-terminus and a $\mathrm{C} 2 \mathrm{H} 2$ or $\mathrm{C} 2 \mathrm{HC}$ zinc motif at the $\mathrm{C}$-terminus of about 60 amino acids in length $[1,6]$. Both the heptapeptide sequence and zinc-finger motif are required for the high binding affinity of WRKY TFs to the consensus W-box (TTGACC/T) cis-elements in their target genes, which regulates target gene expression $[7,8]$. Based on the number of WRKY domains and the structure of their zinc-finger motifs, WRKY proteins can be classified into three main groups: groups I, II, and III. Group II is specified by WRKY proteins containing a single WRKY domain with a $\mathrm{C} 2 \mathrm{H} 2$ motif and can be further divided into five subgroups: IIa, IIb, IIc, IId, and IIe. Group I contains WRKY proteins with two WRKY domains including a $\mathrm{C} 2 \mathrm{H} 2$ motif. The remaining WRKY proteins, containing a WRKY domain along with a C2HC motif, belong to group III $[9,10]$.

The evolution of the WRKY gene family has been studied in a large number of reports, but a clear evolutionary history has so far not yet been defined. This has resulted in an active debate on the origin of individual WRKY genes and gene groups in different plant species $[3,11]$. According to a large phylogenetic tree using higher plants, the WRKY gene family can be classified into four clades, including groups I + IIc, groups IIa + IIb, group IId, and group IIe $[3,12]$. Previous reports have suggested two alternative hypotheses for the evolution of the WRKY family: The "Group I Hypothesis" and the "IIa + b Separate Hypothesis". The "Group I Hypothesis" postulates that the C-terminal WRKY domains of group I genes are the most ancient and that all other WRKY genes are derived from these, whereas the "IIa $+\mathrm{b}$ Separate Hypothesis" suggests that groups IIa and IIb evolved directly from a single domain algal gene, independent from the group I-derived lineage [3, 13, 14].

In addition, WRKY transcription factors are involved in a plant's stress response to herbivores, pathogens, and nematodes $[15,16]$. Substantial evidence has shown that the WRKY TFs play significant roles in the signaling and regulation of gene expression during biotic and abiotic stress responses $[17,18]$. For example, In Camelina (Camelina sativa (L.) Crantz), CsWRKY21 was highly expressed under cold stress and CsWRKY22 was highly expressed under drought stress [17]. In the Grape (Vitis vinifera $\mathrm{L}$.), $V v W R K Y 11$ is involved in the response to dehydration stress, and $V v W R K Y 24$ expression is induced by cold stress [18]. In Cucumber (Cucumis sativus L.), five $C s W R K Y s$ responded strongly to salt stress and hightemperature stress [4]. In Wheat (Triticum aestivum), the expression of TaWRKY75-A was highly induced under salt stress [19]. In Ipomoea trifida (H.B.K.) G. Don, 11 ItfWRKYs were highly expressed under four abiotic stresses: cold, heat, salt, and drought [16]. In Arabidopsis, AtWRKY39 positively regulates the cooperation between the SA and JA activated signaling pathways that mediate the response to heat stress, while AtWRKY 8 is highly expressed in plant roots and significantly upregulated under salt stress [20,21].

Liriodendron, a basal angiosperm genus, resides within the Magnoliaceae family and has diverged into two distinct species: the East Asian (L. chinense (Hemsley) Sargent) and the North-East American (L. tulipifera Linn) 
$[22,23]$. L. chinense is mainly distributed in the south of the Yangtze River, which has further diverged into two separate lineages, the eastern and western groups in China [23]. The flowering mutant gene super long blooming 1(slb1) of L. chinense was identified by transcriptome analysis [24]. There are few reports on abiotic stress research, such as auxin efflux carrier PIN-FORMED $(P I N)$ [25], gibberellin oxidase (GAox) [26], myeloblastosis $(M Y B)$ [27], and C-regeneration binding factors $(C B F)$ gene family [28]. Liriodendron hybrids are intraspecific hybrids crossed by L. chinense and L. tulipfera, which are valuable ornamental tree species in both private and public spaces because of their fast growth, drought tolerance, and strong resistance to diseases and insect pests [29, 30]. Under aluminum (Al) stress, glutamate decarboxylase (GAD) was up-regulated in Liriodendron hybrids, and enhanced the $\mathrm{Al}$ tolerance [31]. Adverse temperature and soil conditions can inhibit the growth of $L$. chinense, such as cold, heat, and drought stress. Knowledge is still lacking on how $L$. chinense deals with abiotic stress at a molecular level. WRKY family is involved in plant growth and development and abiotic stress response and has been reported in many species with available genome sequences. The recently published $L$. chinense genome now provides ample resources with which to carry out bioinformatics-based identification and analysis of WRKY TFs.

In this study, we identified $44 \operatorname{LchiWRKY}$ genes and classified them into three main groups. A comprehensive analysis, including determination of exon-intron structure, motif, gene ontology, gene duplication events, chromosome distribution, subcellular localization, and comparative phylogenetic analysis was performed. Furthermore, global transcriptome expression analysis was performed to identify the gene expression pattern of specific WRKY gene family members under different abiotic stress conditions in L. chinense. In addition, Quantitative real-time PCR (qRT-PCR) was used to verify the expression levels of $L$ chiWRKY genes with different expression patterns. Subcellular localization experiment verified the expression position of LchiWRKY transcription factors. This study provides valuable clues for the functional characterization of the WRKY gene family in L. chinense.

This research reveals the functional roles of the $L$. chinenese WRKY genes under abiotic stress and provides a valuable theoretical basis for increasing forest yield and promoting afforestation of Liriodendron.

\section{Results}

The $L$. chinense genome contains a small number of WRKY genes that vary greatly in length

Since WRKY genes have previously been demonstrated to be involved in abiotic stress response, we set out to characterize the $L$. chinense WRKY gene family. To gain an understanding of the gene diversity present within this family, we determined the basic physicochemical properties of the WRKY protein products, including sequence length, molecular weight (MW), isoelectric point (pI) and other indexes for all 44 identified LchiWRKY (Additional file 1: Table S1). The size of the LchiWRKY proteins varied dramatically, with LchiWRKY37 being the shortest protein at 143 amino acids (aa) in length, whereas LchiWRKY42, the longest, contains 787 aa. Their MW ranges from 16.274 to $90.094 \mathrm{kDa}$, and the $\mathrm{pI}$ from 4.95 (LchiWRKY41) to 9.89 (LchiWRKY36). These data suggest that different LchiWRKY proteins might operate in various microenvironments. The values of the grand average of hydropathicity were negative for all LchiWRKY proteins, indicating that they are all hydrophilic proteins. Almost all LchiWRKYs were defined as unstable proteins, and only 3 LchiWRKYs (LchiWRKY35, LchiWRKY37, LchiWRKY40) with an instability index of less than 40 were considered to be stable proteins.

The number of WRKY gene family in L. chinense is small, and their physical and chemical characteristics vary widely, so it is necessary to explore their taxonomic and phylogenetic characteristics.

\section{The LChiWRKY gene family consists of three distinct phylogenetic groups}

We constructed a phylogenetic tree that would indicate the LchiWRKY protein family's evolutionary interrelatedness. We based our phylogenetic tree on a multiple sequence alignment of the WRKY full length of each protein. We found that the 44 LchiWRKYS are divided into three separate phylogenetic groups: Group I, Group II, and Group III [13], out of which Group II contains five subgroups, being Group IIa, Group IIb, Group IIc, Group IId, and Group IIe (Fig. 1 and Additional file 2: Table S2). The largest subgroup is IIc with 9 members, and the smallest subgroup is IIa with 4 members.

To see how the $L$. chinense WRKY gene family would compare to model plants that each represent a different evolutionary branch, we constructed a phylogenetic tree including the WRKY protein sequences from basal angiosperms, magnolias, monocotyledons, dicotyledons. There are 17 species, including the basal angiosperm Amborella trichopoda, Nymphaea colorata, magnolia Cinnamomum kanehirae, Liriodendron chinense, monocotyledons (Brachypodium distachyon, Oryza sativa, Panicum hallii, Spirodela polyrhiza), dicotyledons (Arabidopsis thaliana, Capsella grandiflora, Cucumis sativus, Eucalyptus grandis, Malus domestica, Gossypium raimondii, Populus trichocarpa, Theobroma cacao, Vitis vinifera). A. trichop$o d a$ is a basal angiosperm, having diverged earlier than $L$. chinense, the others are di- and monocotyledonous plants 


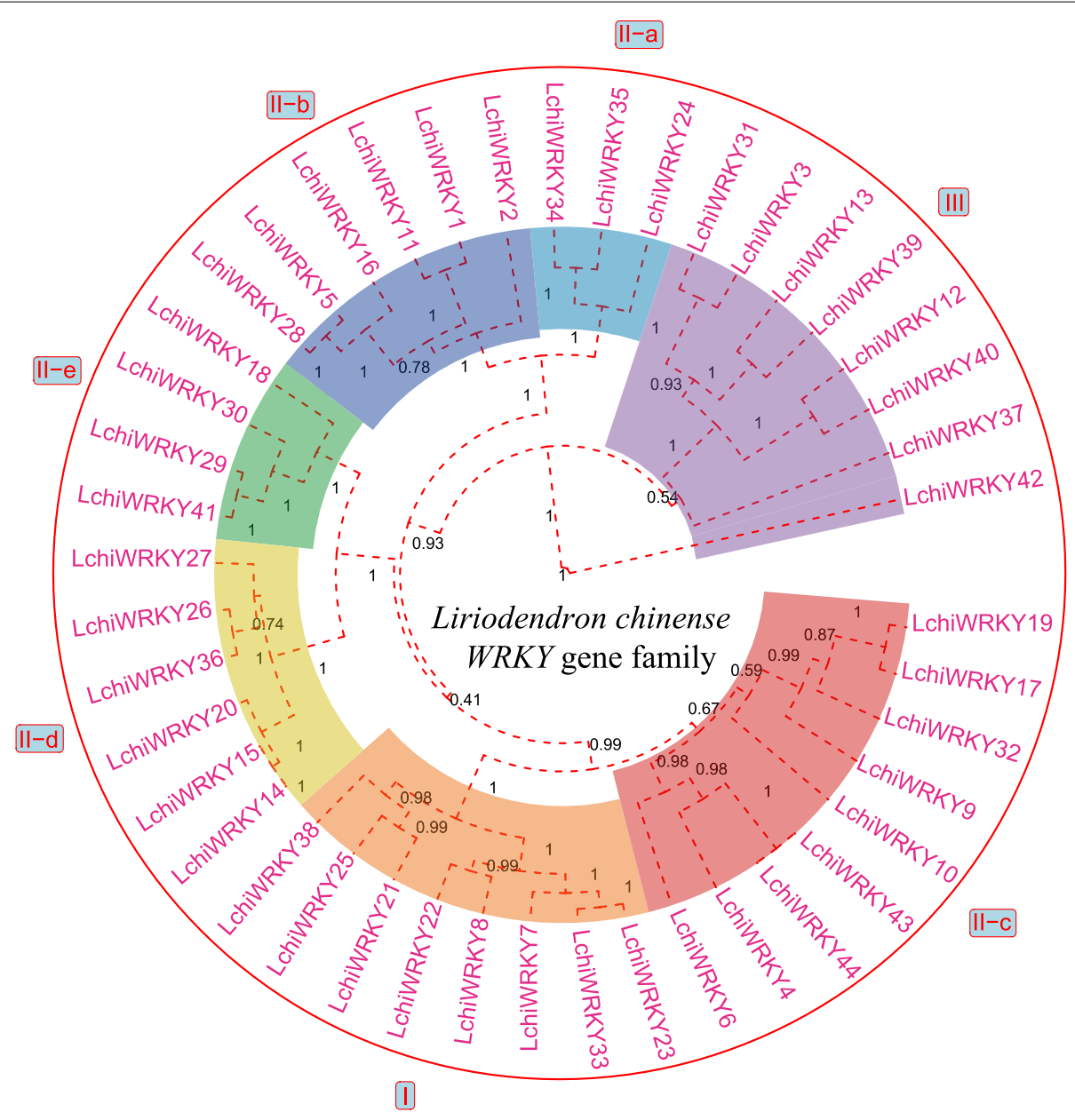

0.3

Fig. 1 Phylogenetic tree of LchiWRKYS. A total of 44 LchiWRKY proteins were aligned using MUSCLE (v3.8.31), and a phylogenetic tree was constructed using BEAST (v2.6.6) set to the Bayesian method with 10,000,000 MCMC chain length. Each color indicates an individual closely related group of WRKY proteins (I-III) within the phylogenetic tree, Posterior probability limit was 1.0 and the posterior value was shown on the branch. The scale bar indicates the branch length of the phylogenetic tree. The posterior value below 0.5 indicates a low homology among the different LchiWRKY protein sequences

respectively that have diverged significantly later than $L$. chinense [23]. A total of 1308 WRKY proteins were used to construct a Bayesian phylogenetic tree as described in the Methods section (Fig. 2 and Additional file 3: Table S3).

All 1308 WRKYs clustered across major clades of the classification group. We frequently found WRKY members from all 17 species that belong to one specific group (I, II, III) to be clustering to the same clade. We found LchiWRKY protein sequences to be more closely related to their homologs from $C$. kanehirae than those from monocotyledons and dicotyledons. This finding is consistent with the evolutionary status of $L$. chinense being closer to $A$. trichopoda, another basal angiosperm, than model plants A. thaliana and O. sativa, two more recently evolved dicotyledonous and monocotyledons species [1].

\section{The LchiWRKY family was at a relatively primitive evolutionary status and had fewer family members}

Interestingly, the total number of WRKY families in the majority of monocotyledons and dicotyledons is more than that in L. chinense (44) and A. trichopoda (31) (Table 1 and Additional file 4: Table S4), for example, among monocotyledons, S.polyrhiza has the least number of WRKY families (44), and among dicotyledons, $V$. vinifera has the least number of WRKY families (60), but most of them were more than that of $L$. chinense. For 


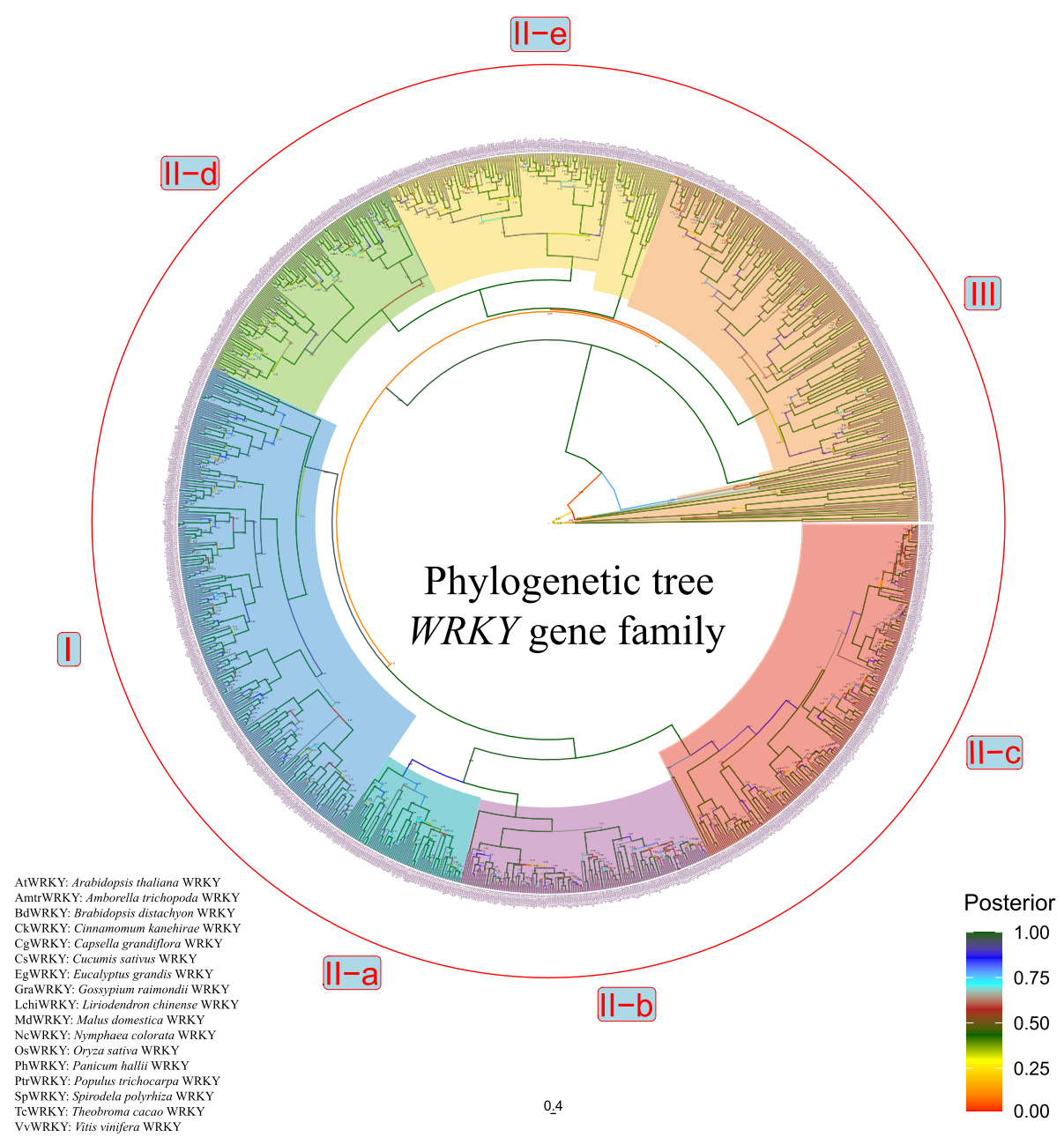

Fig. 2 Unrooted Classification tree representing relationships among WRKY genes of 17 species. A total of 1308 WRKY proteins were aligned by MUSCLE(v3.8.31), and a phylogenetic tree was constructed using BEAST (v2.6.6) set to the Bayesian method with 10,000,000 MCMC chain length. Each label indicates an individual phylogenetic group (I-III). The posterior probability limit was 1.0 and the posterior value was shown on the branch. Different highlighted colors of clades represent the different groups and different branch colors represent the corresponding posterior values. The scale bar indicates the branch length of the phylogenetic tree. The legend indicated the posterior values, which below 0.5 indicate a low homology among the protein sequences involved

Magnolia plants, two whole-genome duplication (WGD) events occurred during the evolution of C. kanehirae [32], and only one WGD event occurred in L. chinense [23], which may lead to a greater number of WRKY genes in C. kanehirae (73) compared to L. chinense (44). As the three subgroups (I, II, II) of plants of different evolutionary statuses are clustered into the same branch, so it is speculated that the WRKY family is relatively conservative in the process of evolution. We infer that different plant subgroups have different numbers due to differential expansion speeds and finally lead to different total family numbers, as a result, the WRKY family number of some basal angiosperms and magnolias expanded slower than monocotyledons and dicotyledons [14].
Then we used the OrthoFinder (v2.2.3) to explore the WRKY evolutionary status of all the 17 species (Fig. 3 and Additional file 4: Table S4), and the phylogenetic analysis showed that the evolutionary status of WRKY of $L$. chinense was closer to the Magnolia C. kanehirae, and the evolutionary status of both species is relatively lower than that of higher angiosperms, including monocotyledons and dicotyledons, while higher than basal angiosperms $A$. trichopoda and N.colorata (Fig. 3). These results suggested that $L$. chinense might experience a slower expansion model during evolution, resulting in a smaller number of family members than other higher plants. So it is necessary to explore the expansion pattern of the $L c h i W R K Y$ gene family. 
Table 1 The total number and subgroups of WRKY families in 17 species

\begin{tabular}{llllllllll}
\hline Taxon & Species & Total_number & Group I & Group Ila & Group IIb & Group Ilc & Group IId & Group Ile & Group III \\
\hline Basal Angiosperms & A.trichopoda & 31 & 7 & 2 & 4 & 9 & 3 & 3 & 3 \\
Basal Angiosperms & N.colorata & 65 & 18 & 6 & 9 & 10 & 7 & 10 & 5 \\
Magnolia & L.chinense & 44 & 7 & 3 & 6 & 8 & 4 & 7 & 9 \\
Magnolia & C.kanehirae & 73 & 15 & 6 & 8 & 12 & 9 & 12 & 11 \\
Monocotyledon & S.polyrhiza & 44 & 6 & 2 & 6 & 11 & 6 & 8 & 5 \\
Monocotyledon & O.sativa & 76 & 16 & 4 & 8 & 10 & 10 & 5 & 23 \\
Monocotyledon & B.distachyon & 89 & 17 & 5 & 5 & 16 & 10 & 10 & 26 \\
Monocotyledon & P.hallii & 120 & 35 & 7 & 16 & 18 & 13 & 17 & 14 \\
Dicotyledon & V.vinifera & 60 & 16 & 4 & 8 & 12 & 6 & 8 & 6 \\
Dicotyledon & T.cacao & 61 & 14 & 3 & 8 & 10 & 6 & 7 & 13 \\
Dicotyledon & C.sativus & 62 & 18 & 4 & 5 & 11 & 8 & 8 & 8 \\
Dicotyledon & C.grandiflora & 69 & 16 & 3 & 8 & 13 & 6 & 8 & 13 \\
Dicotyledon & A.thaliana & 72 & 18 & 3 & 8 & 12 & 7 & 8 & 16 \\
Dicotyledon & E.grandis & 79 & 17 & 7 & 10 & 17 & 8 & 7 \\
Dicotyledon & P.trichocarpa & 102 & 16 & 4 & 8 & 13 & 12 & 11 \\
Dicotyledon & G.raimondii & 102 & 25 & 5 & 9 & 22 & 14 & 16 \\
Dicotyledon & M.domestica & 159 & 32 & 8 & 17 & 29 & 14 & 21 \\
\hline
\end{tabular}

Total_number indicates the total number of WRKY families, Group I III indicates the subgroups number of WRKY families

\section{The LchiWRKY gene family expanded due to gene tandem} duplication events in the evolutionary past

To understand how the identified LchiWRKY genes are distributed over the $L$. chinense chromosomal landscape, we mapped their sequences onto its 19 chromosomes [23] (Fig. 4 and Additional files 5 and 6: Tables S5-S6). Considering the high diversity with the L. chinense WRKY gene family, we asked how then the different genes would be interrelated. Using $L$. chinense genome

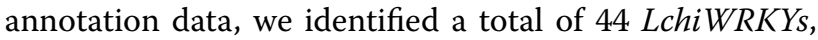
which were renamed from LchiWRKY1 to LchiWRKY4O based on their chromosomal positioning. An additional four genes (LchiWRKY41, LchiWRKY42, LchiWRKY43, and LchiWRKY44) that were mapped to yet unassembled scaffolds were named LchiWRKY41 LchiWRKY 44 respectively.

We found 40 LchiWRKYs to be unevenly distributed across 15 out of the $19 \mathrm{~L}$. chinense chromosomes, with chromosomes 4, 7, 12, and 19 devoid of any WRKY gene. However, the remaining 4 genes mapped onto four separate scaffolds were not yet assembled into full chromosomes. Overall, the number of WRKY genes per chromosome varied from 0 to 5 , with no apparent correlation between chromosomal length and the number of WRKY genes present (Fig. 4A).

Our previous study mapped several gene duplication events that occurred in the evolutionary past of $L$. chinense [23]. Such events could have also affected the number of WRKY genes in the L. chinense genome, as well as their distribution over the genome [3]. Duplication events are known to occur at a regional scale via tandem duplication, copying one gene's sequence in the direct vicinity of the original $(<200 \mathrm{~kb})$, but may also occur at a larger scale via segmental duplication, copying whole sections of chromosomes $[3,13]$. The former is often caused by DNA replication errors, whereas the latter may result from polyploidy events followed by chromosomal rearrangements [3, 33].

We could find a total of 4 WRKY gene pairs on the $L$. chinense genome. Out of these, no gene resulted from a segmental duplication event (Fig. 4A). The 4 events (LchiWRKY27-LchiWRKY15, LchiWRKY27LchiWRKY14, LchiWRKY27-LchiWRKY20, LchiWRKY23-LchiWRKY35) have all likely resulted from tandem duplication, leading to 6 additional WRKY genes (LchiWRKY14,15,20,23,27, and 35) in the L. chinense genome, which distributed on Chr8, 11,14,15, and 16. Two of them were in group I (LchiWRKY23) and IIa (LchiWRKY35), the others were in group IId (Fig. 4B). These results indicate that a few gene duplication events like group IId have indeed contributed to the expansion of the LchiWRKY gene family in the evolutionary past.

To gain an understanding of whether or not the duplicated LchiWRKY genes contribute to organism fitness, we measured the $\mathrm{Ka} / \mathrm{Ks}$ nucleotide substitution ratios of 4 individual gene pairs to study the exerted selective pressure (Table 2).

Generally, a $\mathrm{Ka} / \mathrm{Ks}$ ratio $>1$ is consistent with positive selection, while $\mathrm{Ka} / \mathrm{Ks}<1$ indicates a purifying or 


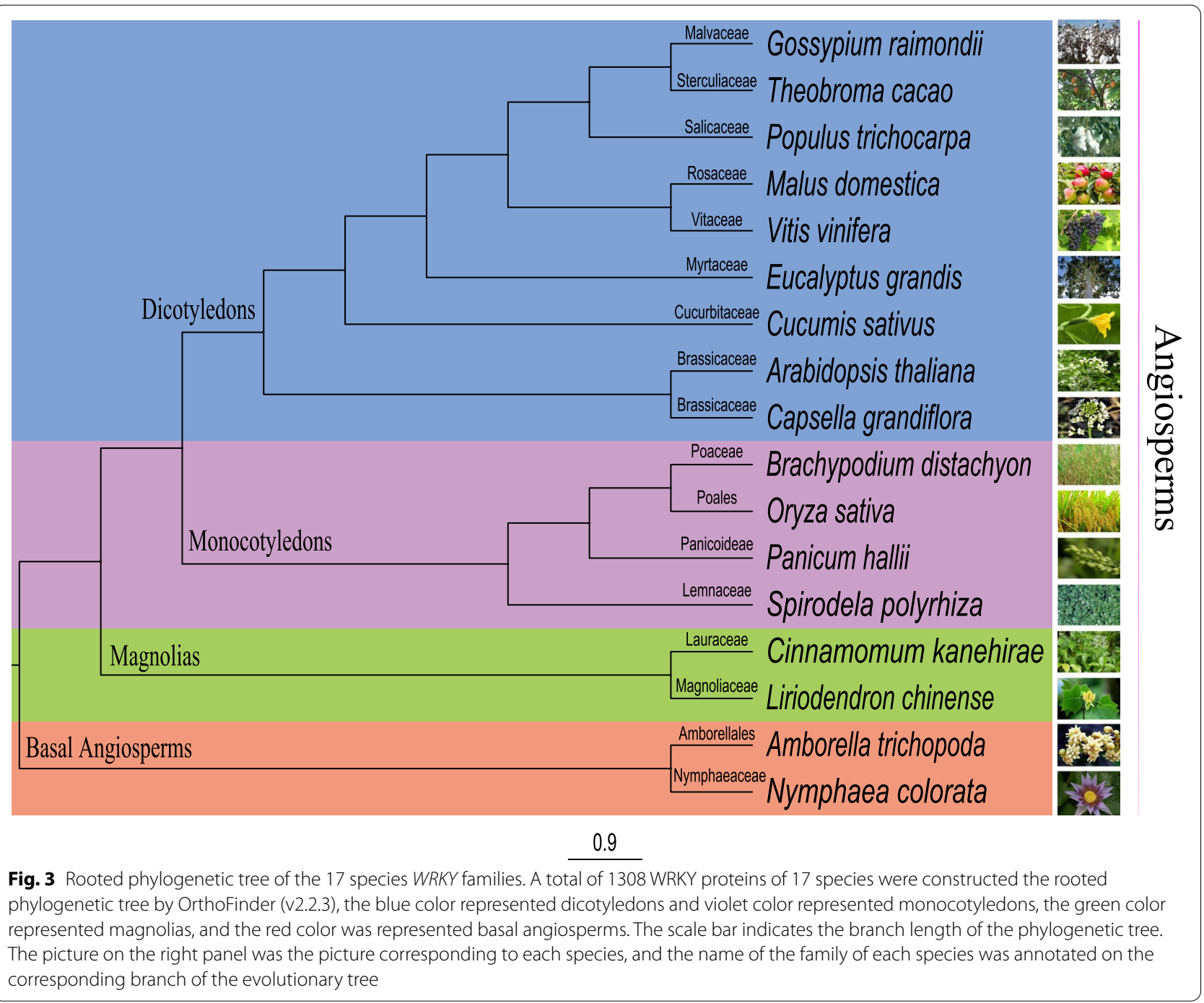

negative selection [34]. We found their $\mathrm{Ka} / \mathrm{Ks}$ ratios all to be $<1$, indicating that the duplicated LchiWRKYs have undergone a synonymous substitution or purifying selection with limited functional divergence during their evolutionary history.

The analysis of gene replication events and selection pressure showed that the WRKY family expanded mainly through tandem duplications, but they were subjected to purifying selection in the process of evolution, so it is necessary to further explore the structural characteristics and functions of LchiWRKYs.

\section{The LchiWRKYs contained multiple motifs and gene ontology enrichment analysis}

To further know the structure of LchiWRKYs and their phylogenetic relationships, we performed a gene sequence analysis for conserved motifs using the MEME suite software package [35], which identified 20 different conserved sequence motifs (motif 1-20) in the LchiWRKY protein family (Fig. 5 and Additional file 7: Table S7).

We found a single motif (motif 1 , a WRKY motif) to be widely distributed among all members of the LchiWRKY family, while all others were specific to one or a few phylogenetic groups we defined previously (Fig. 5). For example, motif 6 (a WRKY motif) and motifs 7, 9, and 19 are only present in Group I members (LchiWRKY38, $25,21,22,8,7,33$, and 23 ), motif 10 and 11 are specific to Groups IIa (LchiWRKY34, 35, and 24) and IIb (LchiWRKY16, 5, 28, 1, 11, and 2), motif 12 to Group IIb, motif 5 to Group IIc (LchiWRKY17, 19, 32, 9, 10, 6, 4, 44 and 43) and I and motif 16, 17 and 20 to Group IId (LchiWRKY26, 36, 27, 20, 15 and 14). Remarkably, Groups IIe (LchiWRKY18, 29, 41 and 30) and III (LchiWRKY40, 12, $39,13,3,31,37$ and 42) did not contain any conserved sequence motifs, suggesting an increased sequence 


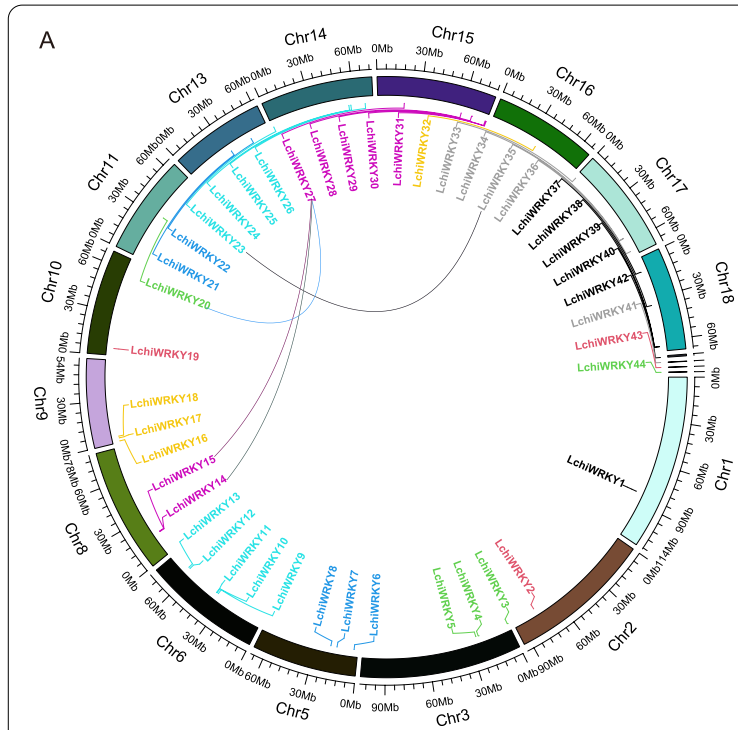

B

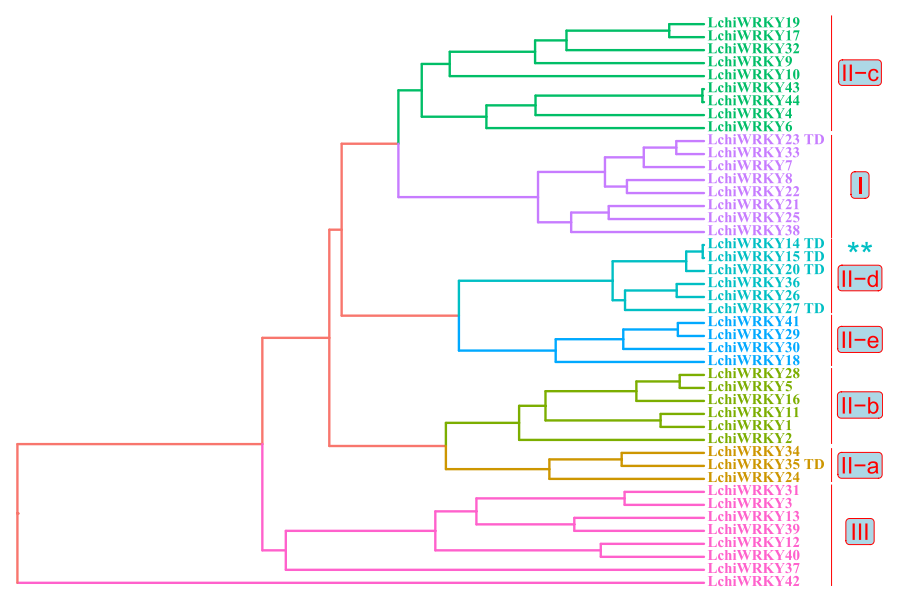

Fig. 4 LchiWRKY chromosomal localization and tandem duplication events in the L. chinense genome. A The chromosomal distribution and positioning of LChiWRKYs are plotted using a Circos plot. The 15 chromosomes that contain WRKY genes (each indicated by a different color) are plotted along with an $\mathrm{Mb}$ (million base pair) scale (1 tick=10 Mb). Individual LchiWRKY gene positions are labeled using the WRKY gene name. Where individual chromosomes (bars) are labeled with respective LchiWRKY genes in the circle, each colored curve indicates the gene duplication event of LchiWRKYs across the chromosomes. B Tandem duplication events distributed across the phylogenetic tree groups. TD indicates a tandem duplication event, each group was labeled with differently colored branches, ${ }^{* *}$ represent the most obvious group of TD event

diversification of these groups (Fig. 5). According to most reports, the functions of some motifs remain to be elucidated. Overall, the closely related LchiWRKYs in the phylogenetic clades shared similar motif compositions, suggesting that the LchiWRKYs within the same phylogenetic group may have similar functions.

To further understand the function of the identified 44 WRKY genes, we performed gene ontology (GO) annotation analysis (Additional file 8: Fig. S1). The results showed that a total of 34 genes were significantly enriched in different GO terms, mainly including 2 categories and 10 subcategories. In the molecular function category, 34 genes were significantly enriched in 3 subcategories, including 'Sequence-specific DNA binding' (GO: 0043565), 'DNA binding transcription

Table $2 \mathrm{Ka} / \mathrm{Ks}$ values of LchiWRKY gene pairs

\begin{tabular}{lllll}
\hline Sequence & Method & Ka & Ks & Ka/Ks \\
\hline LchiWRKY27\&LchiWRKY15 & MA & 0.472679 & 0.988463 & 0.478197 \\
LchiWRKY27\&LchiWRKY14 & MA & 0.472679 & 0.988463 & 0.478197 \\
LchiWRKY27\&LchiWRKY20 & MA & 0.472679 & 0.988463 & 0.478197 \\
LchiWRKY23\&LchiWRKY35 & MA & 0.994488 & 1.10966 & 0.896212
\end{tabular}

The sequences represent duplicated gene pairs, MA represents a model that averages parameters across 14 candidate models, $\mathrm{Ka}, \mathrm{Ks}$, Ka/Ks represent the values of Ka (non-synonymous substitutions per non-synonymous site), Ks (synonymous substitutions per synonymous site), and $\mathrm{Ka} / \mathrm{Ks}$ respectively factor activity' (GO: 0003700) and 'Transcription regulator activity' (GO: 0140110). In the biological process category, they were mainly enriched in 7 subcategories, including 'Regulation of transcription, DNA-templated (GO: 0006355), 'Regulation of nucleic acid-templated transcription' (GO: 1903506), 'Regulation of RNA biosynthetic process' (GO: 2001141), 'Regulation of RNA metabolic process' (GO: 0051252), 'Regulation of biosynthetic process' (GO: 0009889), 'Regulation of macromolecule biosynthetic process' (GO: 0010556) and 'Regulation of cellular biosynthetic process' (GO: 0031326). Molecular functional enrichment is more significant than others, including three terms: DNA binding transcription factor activity, sequence-specific DNA binding, and transcription regulator activity, indicating that these three terms may be the main functions of WRKY genes. The three molecular functional subcategories are more representative than the subcategories of biological processes.

\section{Diversity of gene structure and two main promoters cis-element regulator analysis}

We next examined gene structure by studying the exonintron organization and cis-element in all identified LchiWRKY genes, to gain more insight into the evolution of the WRKY family in L. chinense. Firstly, we could observe a large variation in gene size of different LchiWRKYs, ranging from $525 \mathrm{bp}$ (LchiWRKY37) to 40,981 bp (LchiWRKY25) (Additional file 9: Table S8 and Fig. 6). 


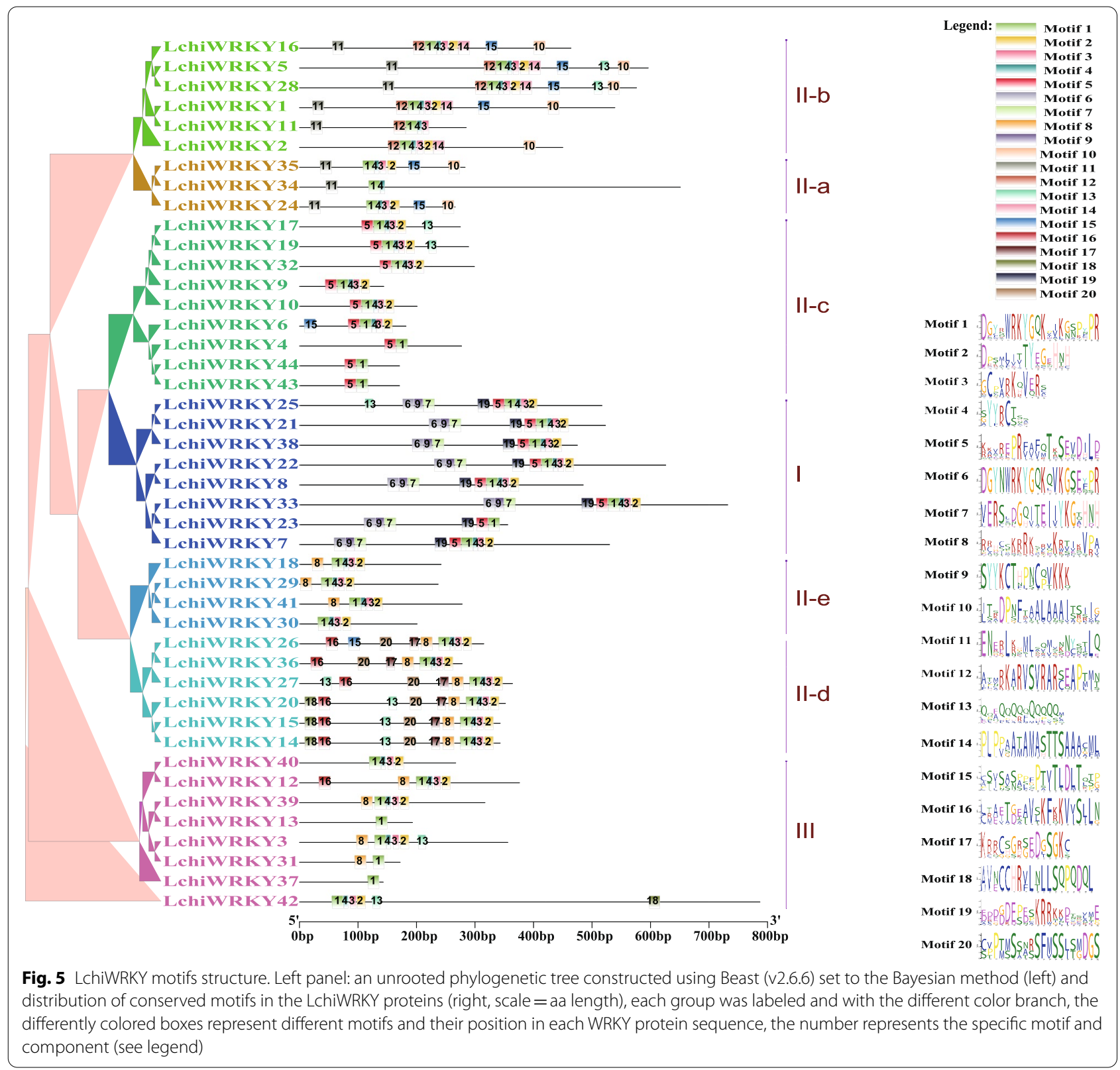

This suggested a large variation in intron length since we had seen previously that the different LchiWRKY CDS sequences were of a much more similar size. The exon number varied between 2 and 6 exons for all LchiWRKY genes, with the majority of genes (27/61.36\%) having a total of 3 exons (Additional file 9: Table S8 and Fig. 6). Two types of introns exist in the conserved WDs. One of the introns is spliced exactly at the $\mathrm{R}$ residue, We classified this phase- 2 intron as an R-type intron. The other is localized before the $\mathrm{V}$ residue, which is at the sixth amino acid after the second $\mathrm{C}$ residue in the $\mathrm{C} 2 \mathrm{H} 2$ motif in the zinc finger region. This phase- 0 intron is designated as a V-type intron. We could identify two major types of introns within the conserved WRKY domains of the LchiWRKY genes (Additional file 10: Fig. S2), which are similar to those conserved in Arabidopsis [13]. Interestingly, phylogenetic groups IIa and IIb possess a V-type, or phase-0 intron, while all other groups (I, IIc-e, and III) contain an R-type, or phase-2 intron (excepting the LchiWRKY42 gene).

The WRKY domain sequence within the $L$. chinense WRKY gene family shows a high degree of conservation (Additional file 11: Table S9), with only two WRKY sequence variants (WRKYGKK and WRKYGKS) present 


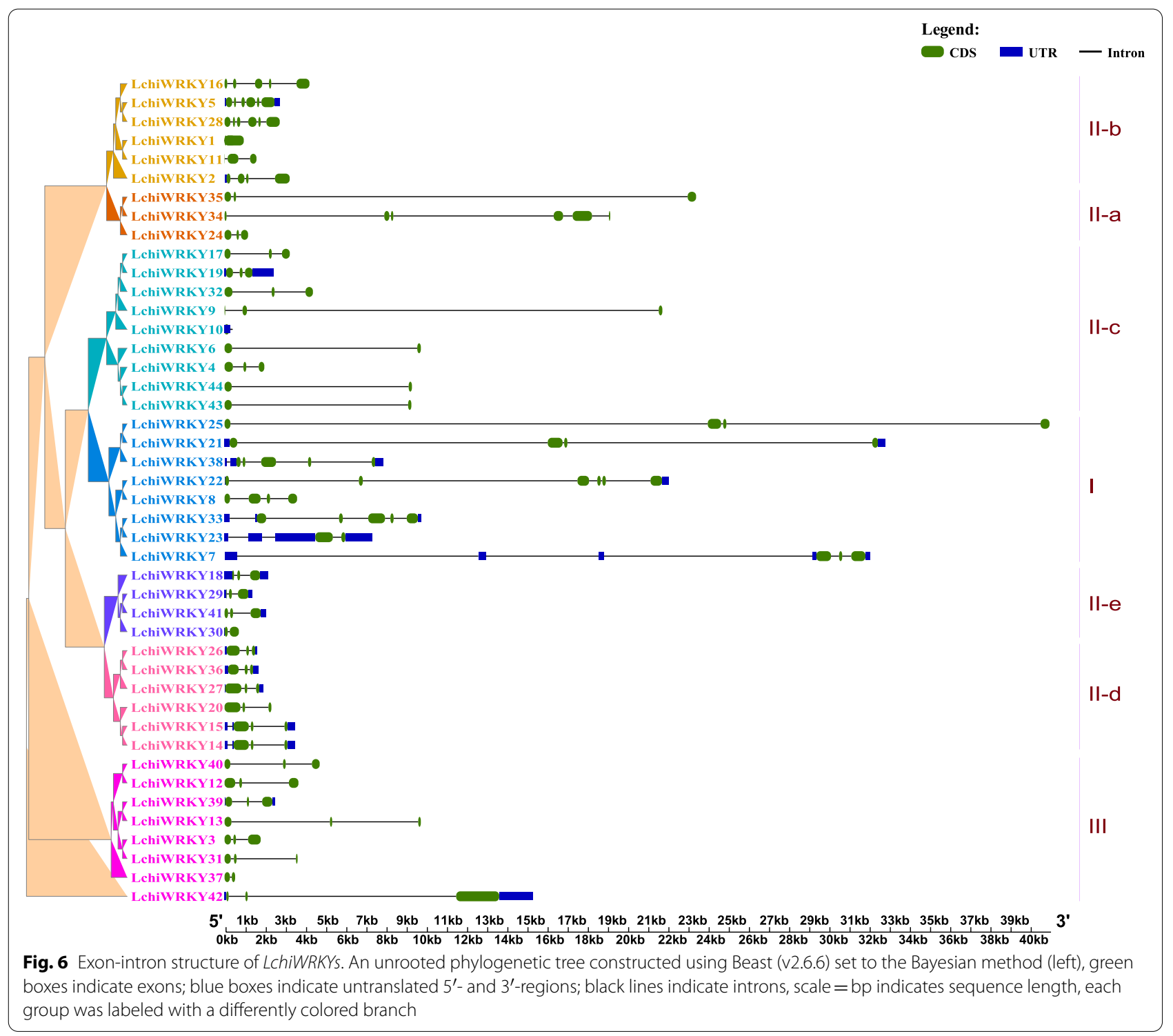

in the LchiWRKY10 and LchiWRKY37 proteins (both in subgroup II-c) respectively. Six proteins, classified in groups II-c and III, lack a C-terminal zinc-motif. Group I contains 8 LchiWRKYs, with each possessing two WDs and $\mathrm{C} 2 \mathrm{H} 2$-type zinc finger motifs (C-X4-C-X22-H-X1-H) (Additional file 11: Table S9). Group II is comprised of 29 proteins, each containing a single WD and $\mathrm{C} 2 \mathrm{H} 2-$ type zinc finger structure (C-X4-5-C-X23-H-X1-H) (Additional file 11: Table S9). We further divided Group II into five subgroups according to their clusters in the phylogenetic analyses, including IIa, IIb, IIc, IId, and IIe with $3,6,10,6$, and 4 members, respectively. Finally, 7 LchiWRKYs with a single WD were assigned to Group III, because of their unique $\mathrm{C} 2 \mathrm{HC}$ zinc-finger structure (C-X7-C-X23-H-X1-C).
We then used the PlantCARE website to analyze $1.5 \mathrm{~kb}$ of the $5^{\prime}$ upstream promoter regions of the LchiWRKY genes to identify any conserved cis-acting elements [4]. Various types of cis-acting elements were found and all Lchi WRKY genes contained several cis-acting elements in their promoter regions. The 12 most common elements are summarized in Fig. 7 (Additional file 12: Table S10). There are two types of cis-elements. One is related to growth and development, including WRKY TF binding element (W-box) and four light-responsive elements (Sp1, Box 4, G-box, and GT1 motif). The other is related to stress response, including abscisic acid-responsiveness (ABRE), MeJA-responsiveness (CGTCA and TGACG motifs), anaerobic element (ARE), drought stressresponsive element (MBS), defense and stress-responsive 


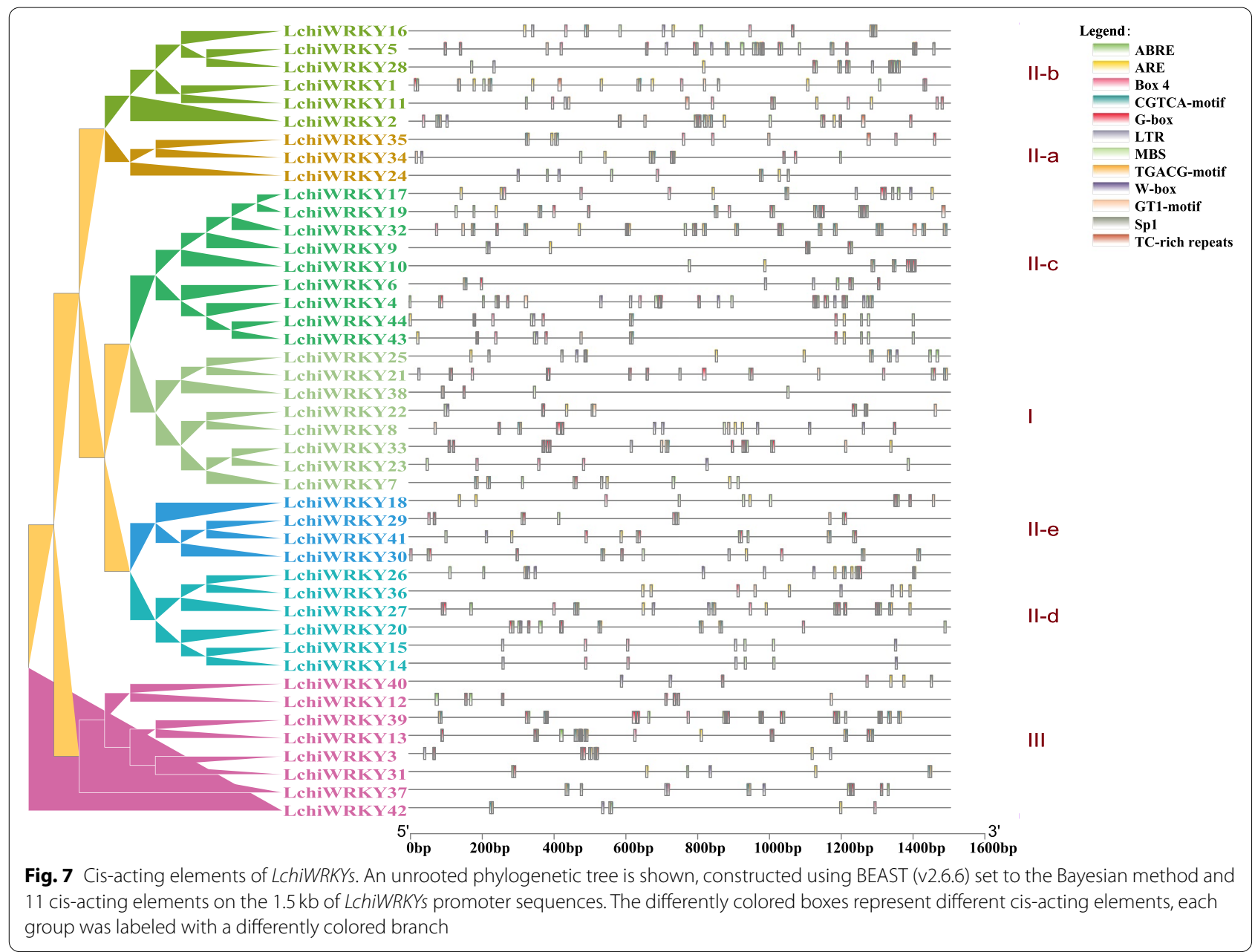

element (TC-rich), low-temperature stress responsive element (LTR). Several of the cis-elements we identified showed a specific distribution across the different $L$. chinense WRKY gene groups. For example, group II-a lacks any LTR elements, suggesting that this group may not be sensitive to low-temperature stress. The Sp1 element can be found in all groups except IId and IIe, showing that the genes in these two groups might not be as responsive to light. Moreover, the TC-rich element could only be detected in groups IIa, IIb, and IIc, indicating that these three groups might be mainly involved in defense and stress response. Finally, the MBS element is not present in group III genes, implying that these genes may not be induced under drought stress. In summary, the $L$. chinense WRKY gene groups seem to possess their own sets of cis-responsive elements, indicating that the different groups may have specialized towards responding to specific sets of stimuli.

Analysis of gene structure, motif and cis-regulatory elements showed that there were two WRKY structural variants and intron insertion structures, $2 \mathrm{GO}$ categories and 12 cis-elements in the WRKY family, which regulated growth and development and abiotic stress response. Therefore, the expression profile of WRKY in growth and abiotic stress needs to be further studied.

\section{The LchiWRKY group II responds strongly to multiple abiotic stresses}

Environmental stress can affect a plant's health and growth, as well as influence the regulation of crucial molecular processes. Under adverse conditions, a large number of stress-related genes are induced to help plants deal with stress. Therefore, to understand how L. chinense deals with abiotic stress and see whether WRKY genes play a role in stress response, as well as their regulatory patterns, we used leaf tissue transcriptome data from 7 subsequent time points $(0 \mathrm{~h}, 1 \mathrm{~h}, 3 \mathrm{~h}, 6 \mathrm{~h}, 12 \mathrm{~h}, 1 \mathrm{~d}$, and $3 \mathrm{~d})$ to explore the expression of $L c h i W R K Y$ genes in response to cold, heat and drought stress (Figs. 8, 9 and 10).

A total of 11 genes were induced by cold stress (Fig. 8 and Additional file 13: Table S11), their expression patterns were clustered into two groups, including persistent 


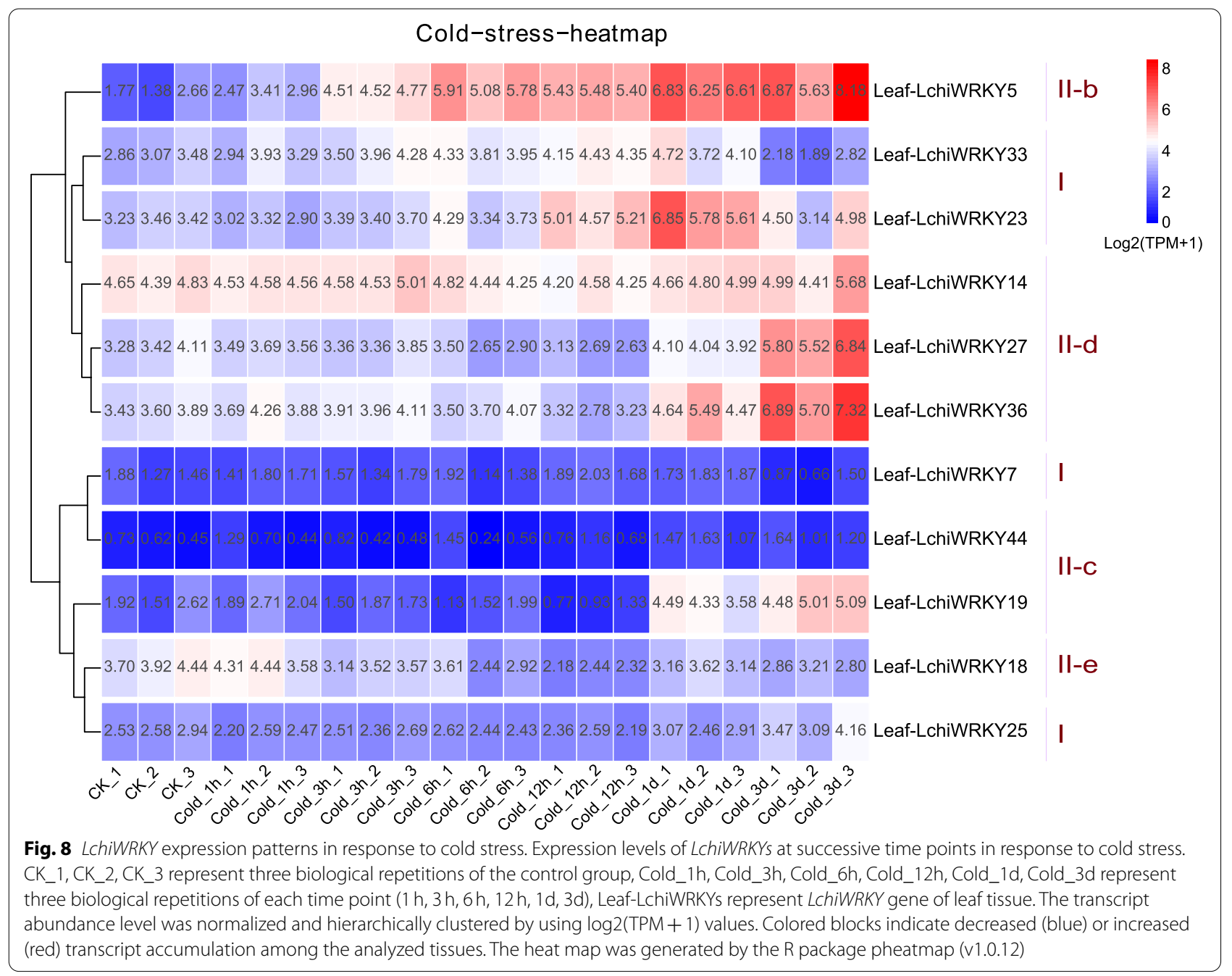

low expression and high expression at different time points. LchiWRKY5, 23, 14, 27, 36, 19 showed peak expression at the $1 \mathrm{~d}$ and $3 \mathrm{~d}$ time points, while downregulated at the other time points. Only two genes showed long-lasting and continuous up-regulation after cold stress, with LchiWRKY5 being up-regulated from $3 \mathrm{~h}$ to $3 \mathrm{~d}$ and LchiWRKY33 from $3 \mathrm{~h}$ to $1 \mathrm{~d}$, LchiWRKY14 was highly expressed at almost all time points. These findings may suggest that LchiWRKY5 and LchiWRKY14 mainly promote $L$. chinense in response to low-temperature stress. $L c h i W R K Y$ family may play a major regulatory role at $1 \mathrm{~d}$ and $3 \mathrm{~d}$ response time points.

The response of LchiWRKY to high-temperature stress was more obvious than that to low-temperature stress, (Fig. 9 and Additional file 14: Table S12). A total of 11 genes were induced by heat stress, and the expression patterns were clustered into two groups, including low expression patterns at almost all time points and high expression patterns at continuous time points $(3 \mathrm{~h} \sim 3 \mathrm{~d})$.
LchiWRKY7 were lowly expressed in all-time points, most of them were differentially expressed in all-time points. LchiWRKY5, 14, 23, 27, 36 were up-regulated significantly at the $1 \mathrm{~d}$ and $3 \mathrm{~d}$ time points, similar to how they responded to cold stress. LchiWRKY44, 18 reached its expression peak at $6 \mathrm{~h}$ and $12 \mathrm{~h}$ time points, while LchiWRKY33, 25 were up-regulated at the $1 \mathrm{~d}$ and $3 \mathrm{~d}$ time points, which were different from cold stress. Compared with cold stress, LchiWRKY18, 25 was specifically high expressed from $1 \mathrm{~d}$ to $3 \mathrm{~d}$ under heat stress. Under heat stress, we speculate that $1 \mathrm{~d}$ and $3 \mathrm{~d}$ may be important response time points.

Under drought stress (Fig. 10 and Additional file 15: Table S13), a total of 13 LchiWRKY family members responded, and their expression patterns were divided into two groups, one group was a high expression from $6 \mathrm{~h}$ to 1 day, the other group was a low expression at all time points. LchiWRKY14 was highly expressed at all time points, It seems that duplication events such as 


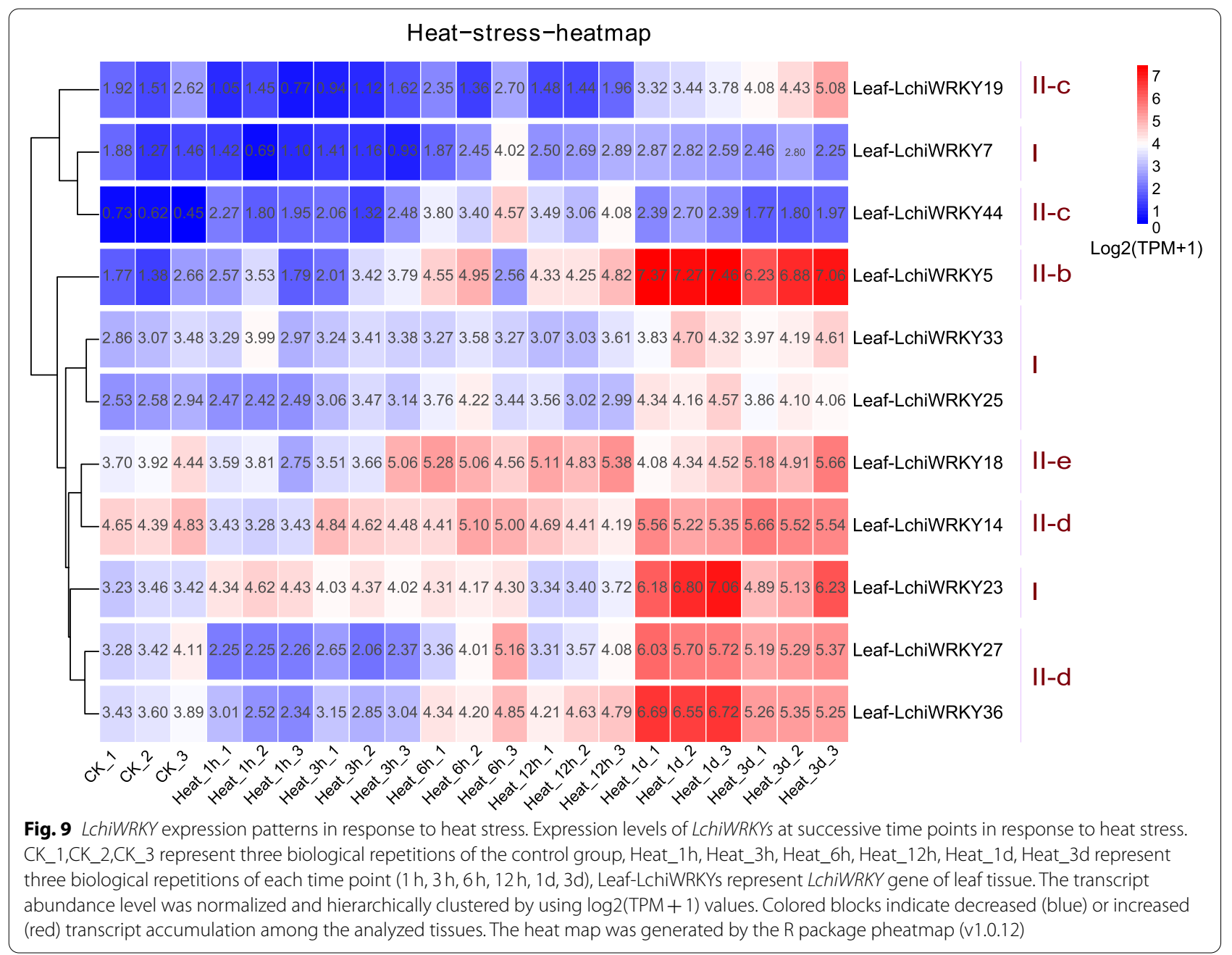

tandem repeat events in the gene structure and promoter region of WRKY family members caused WRKYs to acquire diverse functions $[36,37]$. The expression of LchiWRKY27, 18, 5, 33, 23, 36 peaked at $12 \mathrm{~h}$ and $1 \mathrm{~d}$, and LchiWRKY16, 44, 7, 37, 25, 19 were low expressed at almost all time points. We speculate that $12 \mathrm{~h}$ and $1 \mathrm{~d}$ may be the main response time points, which is different from low and high-temperature stress and may be caused by different biological pathways. LchiWRKY14, 27, 18, 5, 33, 23, 36 may be the main response genes to drought stress.

We found that some genes responded to low temperature, high temperature, and drought stress at the same time, for example, LchiWRKY5, LchiWRKY23, LchiWRKY14, LchiWRKY27, LchiWRKY36 were up-regulated at the $1 \mathrm{~d}$ time points in three cases, suggesting that these five genes are the main responders to abiotic stress and play across regulatory role in abiotic stress resistance, and 1d may be important time points for LchiWRKY to respond to abiotic stress.

\section{The qRT-PCR validation of LChiWRKY under multiple abiotic} stresses

To verify the accuracy of abiotic stress transcriptome data, we performed qRT-PCR experiments of 10 genes with different expression levels, including high or low expression, LchiWRKY7 and 44 were low expression $(0<\log 2 \mathrm{FC}<3)$ in the three stresses and other genes (LchiWRKY5, 18, 19, 25, 27, 33, and 36) were high expression $(\log 2 \mathrm{FC}>3)$ at different time points of the three stresses [38] (Figs. 8, 9 and 10).

Under cold stress (Fig. 11 and Additional file 16: Table S14), LchiWRKY7, 36, 19 were up-regulated and reached the peak at $1 \mathrm{~d}$, while $L c h i W R K Y 23$ was up-regulated and reached the peak at the $12 \mathrm{~h}$ time point, only LchiWRKY5 were up-regulated at all time points. And other genes' patterns like LchiWRKY25, 44, 18, 27, 33 were down-regulated at all time points. As a result, these genes' expression patterns as the same as transcriptome data, and $1 \mathrm{~d}$ was the main response time point. 


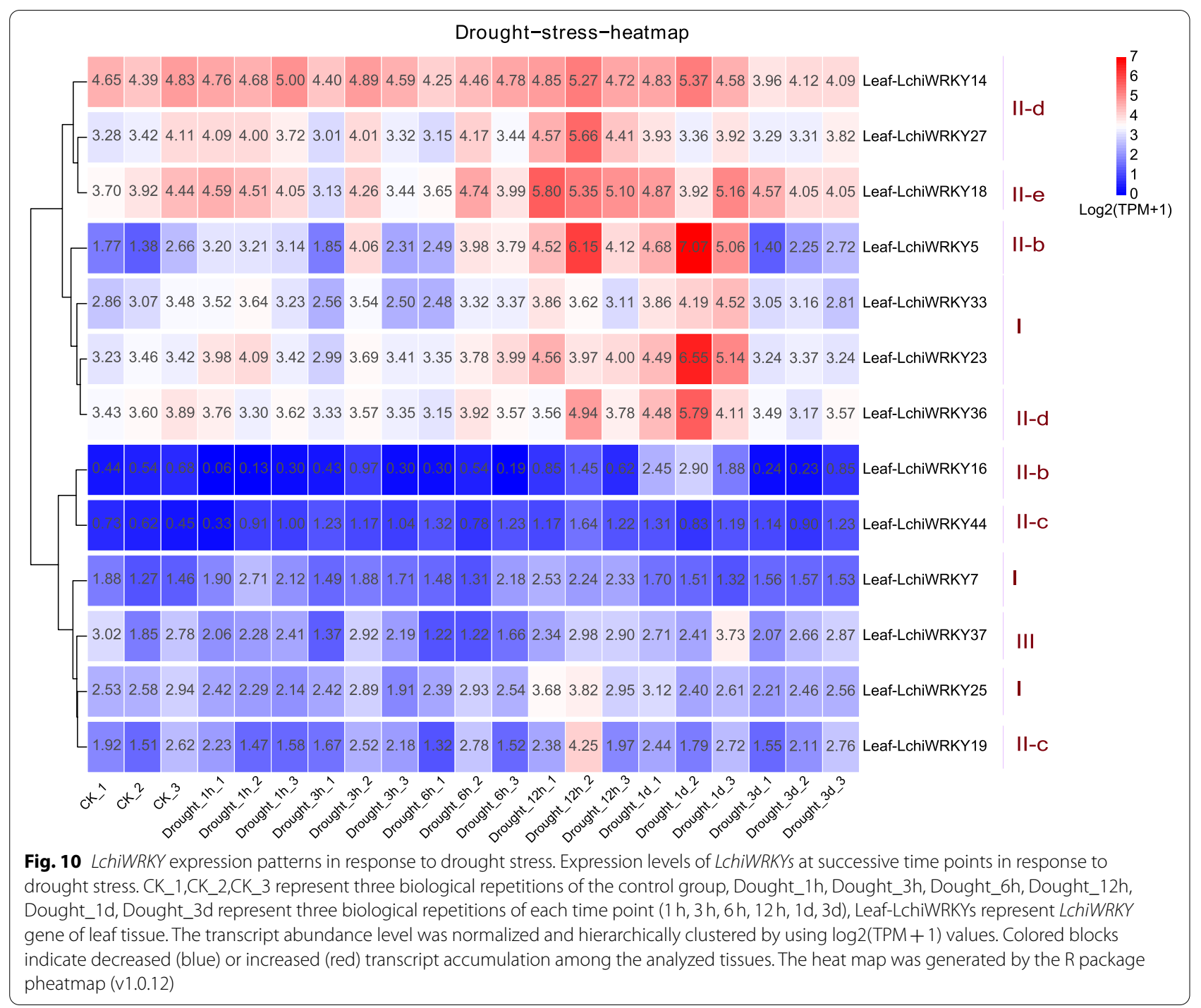

Under heat stress (Fig. 12 and Additional file 17: Table S15), the response time was earlier than that of low-temperature stress. LchiWRKY19 was up-regulated at $3 \mathrm{~h}$ and reached the peak, then LchiWRKY7, 25 were up-regulated and reached the peak at $12 \mathrm{~h}$. The peak expression of LchiWRKY36, 5, 33, 23 were at $1 \mathrm{~d}$, while LchiWRKY44, 27, 18 were low expressed at all time points. The above results showed that the expression pattern of high-temperature stress was different from that of low-temperature stress, but there was a common important response time point $1 \mathrm{~d}$, which indicated that this time point was the key time point for Liriodendron to respond to temperature stress.

Under drought stress (Fig. 13 and Additional file 18: Table S16), the expression of most genes was relatively

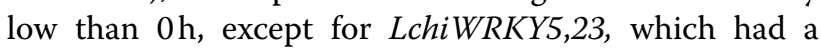
significant peak value and were highly expressed at $12 \mathrm{~h}$ and $1 \mathrm{~d}$, respectively. The response of LchiWRKY18, 19, 33 to drought stress was earlier, peaked at $1 \mathrm{~h}$, and then began to decline. LchiWRKY25, 7, 44, 36, 27 were all low expressed after $0 \mathrm{~h}$, which may be due to the inhibition of drought stress. Compared with temperature stress, the expression levels of some genes were relatively low under drought stress. For instance, LchiWRKY7, 36 was highly expressed under both low and high-temperature stress, but low expressed under drought stress. Several genes had peak expression under three stresses, such as the expression of LchiWRKY5, 23 peaked at $12 \mathrm{~h}$ and $1 \mathrm{~d}$ of three stresses, respectively.

The expression pattern of transcriptome and qRT-PCR experiment of $L c h i W R K Y$ gene under abiotic stress fully showed that the WRKY family of $L$. chinense responded to abiotic stress, which provided the theoretical basis 


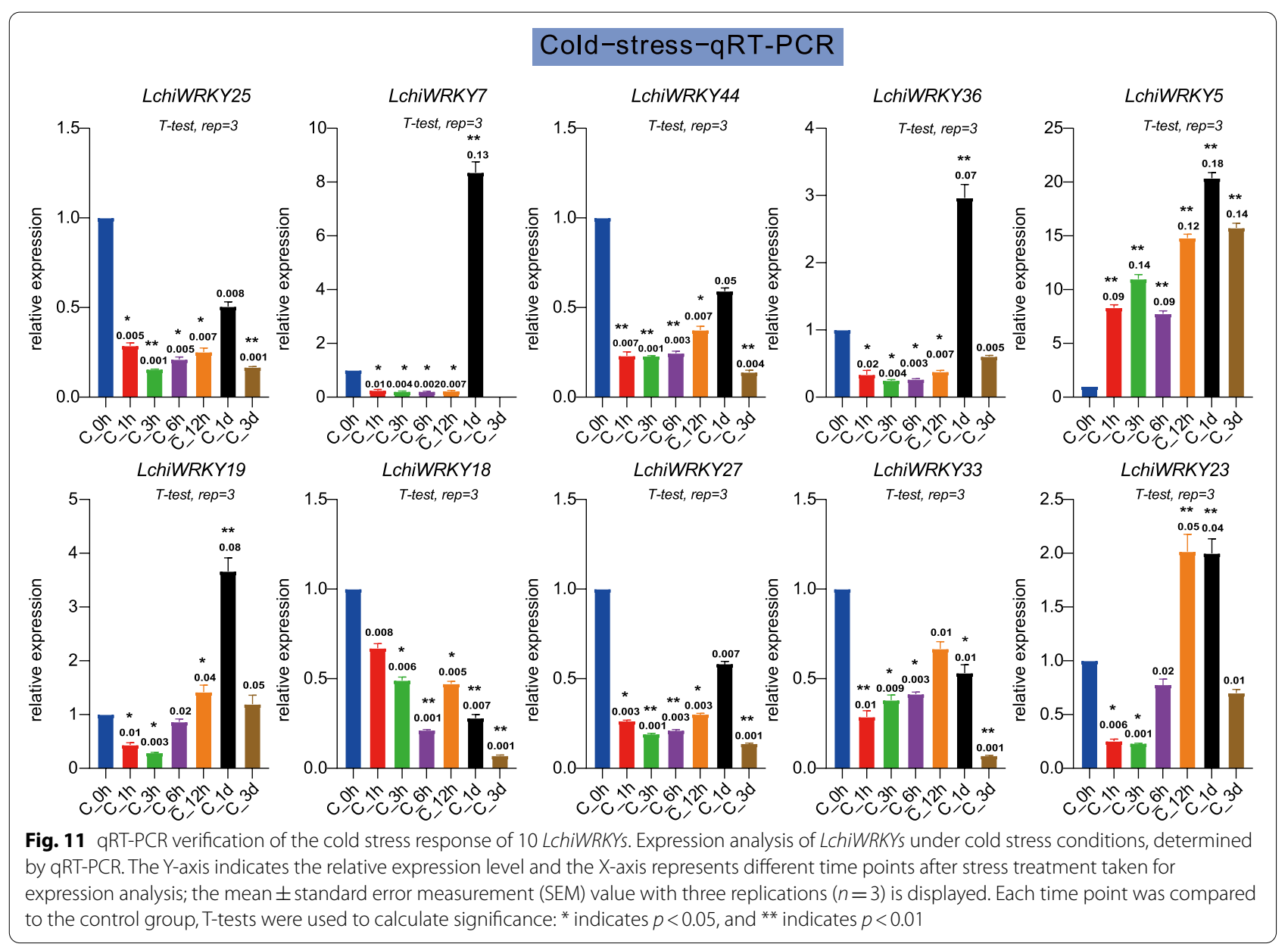

for the adaptability promotion of $L$. chinense in different regions.

\section{LChiWRKY18 and 36 were expressed in the nucleus}

To understand the biological function of WRKY proteins, we performed subcellular localization analysis (Additional file 1: Table S1). The results showed that 44. LchiWRKY genes were expressed in the nucleus [4, 39, 40], while only LchiWRKY42 was also expressed in the cell membrane $[39,41]$. These results suggest that most LchiWRKY transcription factors were expressed in the nucleus, but may also function in other organelles, such as cell membrane. Therefore, we speculate that transcription factors mainly perform biological functions in the nucleus and regulate the expression of target genes in combination with the promoter sequence of target genes. There may also be some other situations, for example, some transcription factors (LchiWRKY42) may also have biological functions in the cell membrane.

To verify the results of subcellular localization analysis, we selected two genes LchiWRKY18 and LchiWRKY36 for the subcellular localization experiment.
The fusion vectors pJIT166-LchiWRKY18-GFP and pJIT166-LchiWRKY36-GFP were constructed and transformed into callus protoplasts of Liriodendron hybrids by PEG-6000 mediated. The subcellular localization expression vector pJIT166-GFP was constructed as a positive control. The fluorescence of LchiWRKY18-GFP (35S::LchiWRKY18::GFP) fusion protein was preferentially detected in the nucleus, while the spontaneous fluorescence of the positive control pJIT166-GFP (35S::GFP) fusion protein could be detected in the whole-cell space (Fig. 14). Similarly, the fluorescence of LchiWRKY36-GFP (35S::LchiWRKY18::GFP) fusion protein was also preferentially detected in the nucleus. The results of the subcellular localization experiment show that LchiWRKY18 and 36 are expressed in the nucleus, which is consistent with the results of subcellular localization analysis, indicating that the main biological functional position of transcription factors is in the nucleus to regulate the transcription of target genes. 


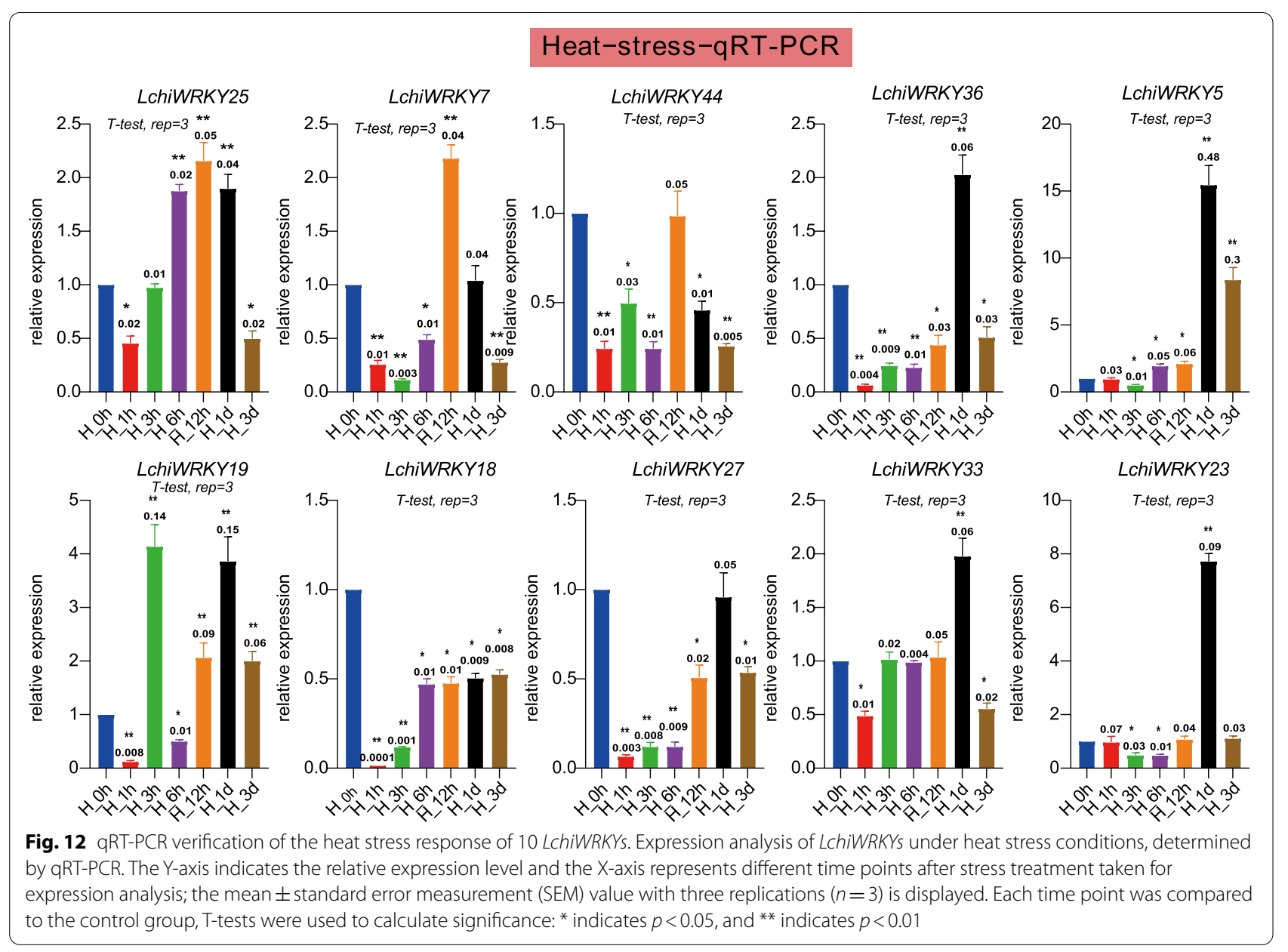

\section{Discussion}

Lchi has fewer WRKY members, diversified biological functions and physicochemical properties, and conserved WRKY domain and motif structure

WRKY genes comprise a large family of transcription factors that are conserved across the whole plant kingdom. Genome-wide analysis of WRKY gene families has been widely carried out in a large number of species whose genomes have been sequenced $[9,42]$. In the current study, a search for WRKY genes in the L. chinense genome resulted in the identification of 44 members, which we designated as LchiWRKY1 through LchiWRKY44, based on their chromosomal location. Compared with some higher angiosperms, the LchiWRKY family has a smaller number. For example, there are $125 \mathrm{ZmWRKY}$ genes in the maize (Zea mays) genome [43], $108 \mathrm{HbWRKY}$ genes in the Rubber Tree (Hevea brasiliensis) genome [44], 102 LuWRKY genes in Flax (Linum usitatissimum L.) genome [45], 86 HvWRKY genes in Barley (Hordeum vulgare L.) genome [46], and 64IiWRKY genes were identified in the whole genome of Isatis indigotica [47]. A total of 61
CsWRKY genes in the Cucumber (Cucumis sativus L.) genome [4], $57 C m W R K Y$ genes were identified in the melon (Cucumis Melo L.) genome [48], and 54 AcWRKYs in Pineapple (Ananas comosus (L.) Merr.) genome [49]. Although the number of WRKY members of these plants with different evolutionary statuses is different, they are more than $L$. chinense. The results showed that the number of WRKY family members of $L$. chinense might be related to its evolutionary status and family expansion model, $L$. chinense was close to the basal angiosperm [23], so speculate the number of LchiWRKY family members might be less than that of higher angiosperm plants.

Gene ontology (GO) showed that most WRKY genes were enriched in molecular function, biological process, and cellular component. For instance, GO analysis of Tartary buckwheat WRKYs showed that most FtWRKYs are enriched in molecular function and biological processes, and some genes are enriched in a cellular component [41]. In Orchardgrass, $93 D g W R K Y s$ were enriched in biological regulation and cellular process of biological process category, and binding and nucleic acid binding 


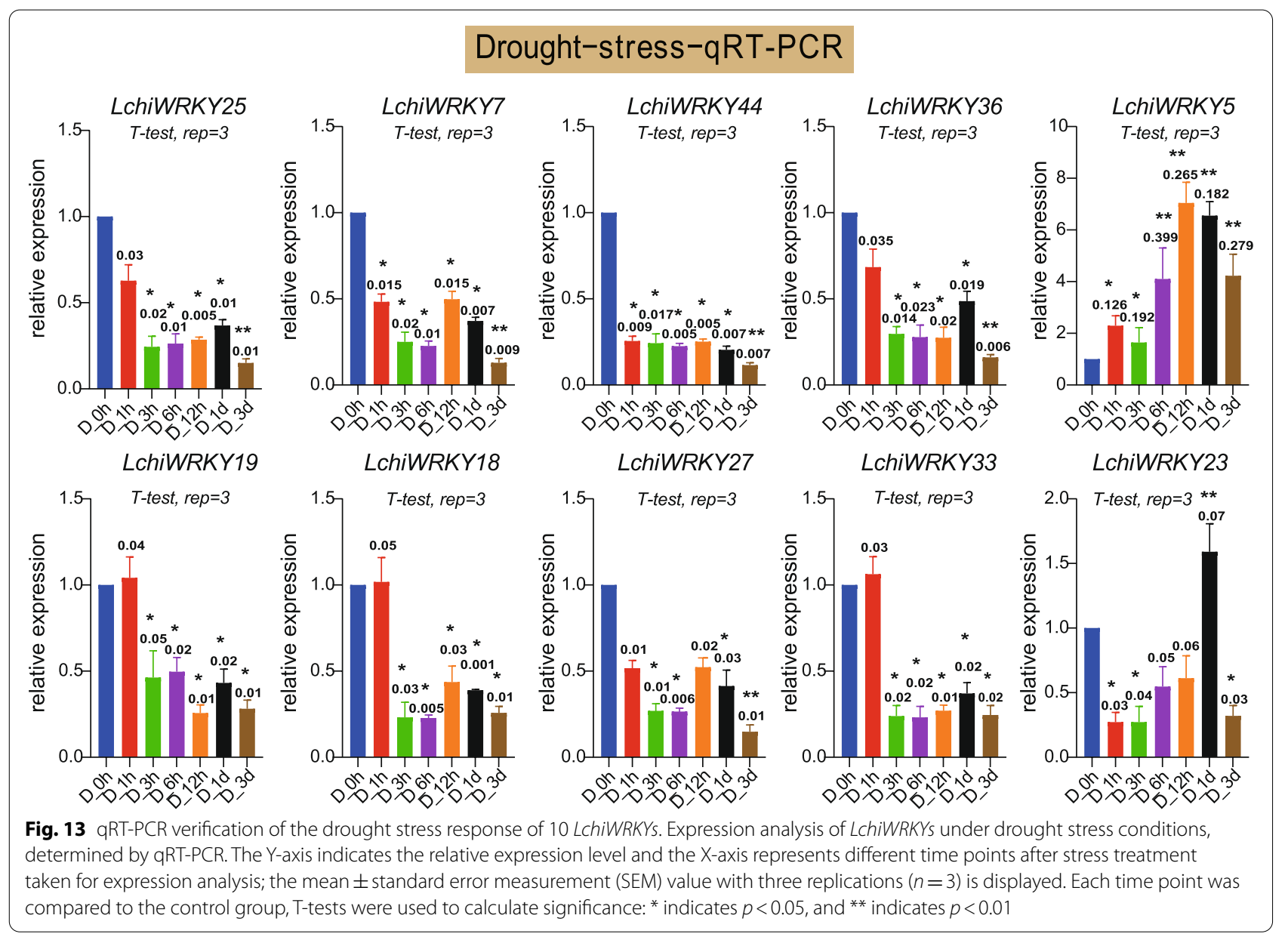

TF activity of molecular function category, while some of them were in membrane and membrane part of cellular component category [50]. In Rice, novel candidate OsWRKY genes were significantly enriched in sequencespecific, DNA, heterocyclic and regulatory region binding activities of molecular function [51]. In Taraxacum antungense Kitag, 44 TaWRKYs were significantly enriched in 23 terms of biological process, and cellular processes were the most representative [52]. In $L$. chinense, A total of 34 LchiWRKYs were significantly enriched in the two GO categories of biological processes and molecular functions, including 10 subcategories, which do not contain cellular components. This is different from the above species. It is speculated that it may be the difference in the regulatory function of WRKY transcription factors among different species. Therefore, biological processes and molecular function may be the main biological functions and processes involved in LchiWRKYs.

The protein length (MW) and isoelectric point (pI) of LchiWRKY proteins showed a large degree of variation across the gene family, indicating that LchiWRKYs possess different protein structures and may have adapted to different microenvironments. These features suggest that LchiWRKYs may have biological regulatory functions in different environments/conditions, especially during abiotic stress. The WRKY protein length, molecular weight, and isoelectric point are different in different species, for example, in Pineapple, the protein length of AcWRKYs varied from 122 to 1320 amino acid (aa), and MW varied from 13.7 to $144.5 \mathrm{kDa}$, and $\mathrm{pI}$ ranged from 5.11 to 10.08 [49]. In Maize, the length of ZmWRKYs varied from 99 to $729 \mathrm{aa}$, and MW varied from 11.27 to $78.7 \mathrm{kDa}$, and $\mathrm{pI}$ ranged from 4.55 to 10.78 [43]. Therefore, the biological functions of WRKY genes in different species are different, which may be related to the physical and chemical properties and spatial structure of proteins.

The conserved WRKY domains of the LchiWRKY proteins were assessed in this study. Multiple sequence alignments revealed that two LchiWRKY proteins (LchiWRKY10 and LchiWRKY37) in group IIc had sequence variation in their WRKY domain. Most characterized WRKY proteins exhibit a binding preference to their cognate cis-acting W-box element, with the help of the WRKY domain. According to previous studies, variations 


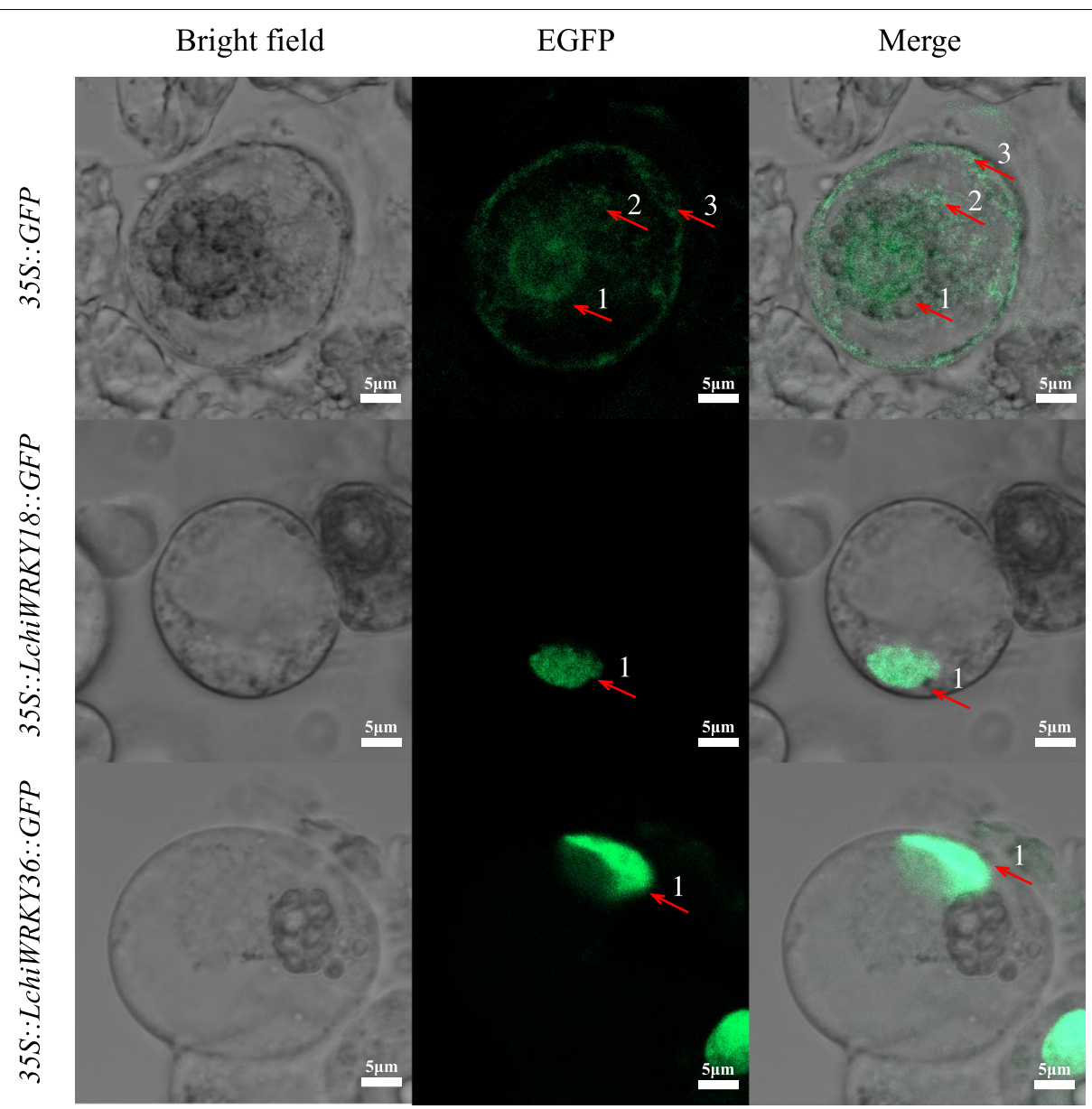

Fig. 14 Subcellular experimental results of LChiWRKY18 and 36. Callus protoplasts of Liriodendron hybrids are used to capture bright field, green fluorescence, bright field, and green fluorescence fusion images. Red arrows 1, 2, and 3 represent the nucleus, cytoplasm, and cell membrane, respectively. Bright field indicates bright field photography, EGFP indicates green fluorescence photography, and Merge indicates the fusion of bright field and green fluorescence. Scale bar $=5 \mu \mathrm{m}$. 35S::: GFP indicates the empty vector pJIT166-GFP, 35S::LChiWRKY18::GFP represents recombinant vector pJIT166-LChiWRKY18-GFP, and 35S::LchiWRKY36::GFP represents recombinant vector pJIT166-LChiWRKY36-GFP

in the WRKYGQK motif within the WRKY domain might influence normal interactions of WRKY genes with their downstream target genes, and it might therefore be worthwhile to further investigate the binding specificity and functionality of these two WRKY proteins $[53,54]$. R-type introns (PR intron) and V-type introns (VQR intron) are two major introns within the WRKY family in different plants; In L. chinense, the groups I, IIc, IId, III we identified contain R-type introns, and groups IIa and IIb contain V-type introns $[4,55]$. These two types of introns may play important roles in biological processes, such as heat stress, cold stress, salt stress, and drought stress. For example, LchiWRKY5 and LchiWRKY23 were highly expressed under cold, heat, and drought stress [4, 11, 55]. These WRKY protein variants and two intron insertions have different forms in different species, which indirectly reflects the conservation and diversity of $W R K Y$ gene in the process of plant evolution, so that the functions of WRKY gene among different species are not only similar but also different $[4,11,54,55]$.

The conserved motifs reveal the grouping specificity of motif distribution of L. chinense, a total of 20 motifs were distributed in 7 subgroups, each of which had a specific motif, except for groups IIe (LchiWRKY30) and III (LchiWRKY40, LchiWRKY42). In Orchardgrass, 10 motifs were determined and the same phylogenetic group has the same conserved motif, only group III didn't have a $\mathrm{C} 2 \mathrm{H} 2$ zinc finger motif [50]. In Common walnut, 15 motifs were detected in JrWRKYs, only two genes contain the CCCC domain, while other phylogenetic subgroups contain group-specific motifs [40]. The above results show that although the number of WRKY 
is different in different species, the motif has specificity in different phylogenetic groups, and the same subgroup has the same motif.

\section{The expansion of the LchiWRKY family results from tandem repeat events and functional and structural diversity of replication genes}

Tandem and segmental duplication events have played a critical role in the expansion of the WRKY gene family [6]. In Rubber tree (Hevea brasiliensis), among the 25 pairs of duplicated gene pairs, 2 pairs of genes were derived from tandem duplication events and 23 pairs of genes were segmental duplication events [44]. In Quinoa (Chenopodium quinoa Wild.), a total of 39 pairs of replication gene pairs, 2 pairs of genes are tandem replication, 37 pairs of genes are segmental replication [14]. In Cucumber (Cucumis sativus L.), 14 segmental replication events occurred in 25 genes [4]. In Pineapple (Ananas comosus), 7 pairs of genes were tandem duplication genes and 17 segmental replication events occurred in 27 genes [49]. In Maize (Zea mays), 52 segmental duplication events occurred in 78 genes, and there was no tandem replication event [43]. In Barley (Hordeum vulgare L.), 5 tandem duplication events and 13 pairs of genes were segmental duplications [46]. In Chinese woad (Isatis indigotica), 5 gene pairs were tandem duplication and 7 gene pairs were segmental duplication [47]. In addition, whole-genome duplication events may also affect the number of gene families. For basal angiosperms, for example, there are no ancestor whole-genome duplication events in A. trichopoda [32], while there is an ancestor whole-genome duplication events in Water lily [56], which may lead to the total number of WRKY in water lily being more than that in A. trichopoda (Table 1 ). For Magnolia plants, two whole-genome duplication events occurred in C. kanehirae and one whole-genome duplication event occurred in L. chinense [23, 32], which may also lead to more WRKY in C. kanehirae than L. chinense (Table 1).

A comparison of the number of WRKY genes in $L$. chinense with other sequenced higher angiosperm genomes shows that L. chinense possesses a smaller number of WRKY genes. This could indicate a reduced number of duplication events in L. chinense, only six genes had tandem replication events. Among these genes, four (LchiWRKY14,15,20,27) are from group II-d, and the other two are from group I (LchiWRKY14) and II-a (LchiWRKY35), This suggests that group IId may play a major role in the expansion of LchiWRKY family, and tandem repetition is the main expansion model. Therefore, the total number of gene families may be related to wholegenome duplication events, tandem duplication, and segmental duplication events, which ultimately determines the difference in the number of WRKY families in different species.

We found that the expression pattern of duplicated paralogous $L$ chiWRKY genes was always different. Divergence of gene expression plays an important role in the preservation of duplicated genes $[57,58]$. Several paralogous gene pairs show a differing response to abiotic stress, suggesting that they may play diverse roles in $L$. chinense in response to abiotic stress (Figs. 8, 9 and 10). For instance, LchiWRKY23 was highly expressed in response to cold and drought stress at $12 \mathrm{~h} \sim 1 \mathrm{~d}$, while its paralogous gene LchiWRKY35 was not induced upon cold and drought stress. Still, some WRKY genes and their paralogues, like LchiWRKY14 and LchiWRKY27, shared similar high transcript abundance profiles under heat stress at $1 \mathrm{~d}, 3 \mathrm{~d}$, suggesting that they may have redundant functions. Further analysis showed that the duplicated genes contained the same cis-regulatory elements (Fig. 7), such as LTR elements, which mainly responded to lowtemperature stress, so they could all respond to lowtemperature stress, such as LchiWRKY27,14. Combined with the results of gene structure and motif, these replicated genes also have diversity in structure and motif. For instance, LchiWRKY23 has 6 motifs and LchiWRKY35 has 7 motifs, only motif 1 is their common motif (Fig. 5). LchiWRKY27 lacks motif 18, and LchiWRKY14, 15 and 20 have it (Fig. 5). LchiWRKY35 has 3 exons and LchiWRKY23 has 2 exons and 4 UTRs, but no UTR is found in LchiWRKY35 (Fig. 6).

\section{LchiWRKY gene has a cross-regulation function in response to multiple abiotic stress}

According to previous studies, WRKY transcription factors are involved in abiotic stress regulation, including low temperature, high temperature, salt, and drought [4, 16, 44]. For instance, in Ipomoea trifida, 11 Itfwrkys (Itfwrky8, 15.1, 22.1, 34, 41, 48, 66, 69, 77, 79, and 80 ) were highly expressed under salt, drought, low temperature, and high temperature [16]. In Cucumber (Cucumis sativus L.), 21 CsWRKY genes were induced to express under heat stress, and the expression trend was the same from $3 \mathrm{~h}$ to $6 \mathrm{~h}$, five $C s W R K Y$ genes (CsWRKY27 / 41 / 50 / 52 / 57) were induced to express under salt and heat stress [4]. In Camelina (Camelina sativa (L.) Crantz), the expression of CsWRKY21 under cold stress was higher than that under drought stress, while the expression level of CsWRKY22 under drought stress was higher than that under cold stress [17]. In Quinoa (Chenopodium quinoa Wild.), nearly $46 \mathrm{CqWRKY}$ genes are induced to express under salt or drought stress. The expression level of CqWRKY52a-1 / 2 was similar to that of group II-d, which was highly expressed under salt stress [14]. In Chickpea (Cicer arietinum L.), 
15 genes were down-regulated in roots and 10 genes were down-regulated in stems under abiotic stress [59]. The OsWRKY55 negatively regulates drought tolerance in transgenic rice lines [60], The transcription level of HvWRKY42 increased in Barley (Hordeum vulgare L.) under drought stress, which indicated that $H v W R K Y 42$ was regulating the drought adaptability of barley [61]. The $H \nu W R K Y 3$ showed 13 fold high expression and 4 genes showed 2-fold high expression under drought stress [46].

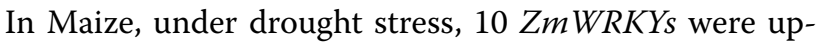
regulated in roots and $4 Z m W R K Y s$ showed 10-folds expression in leaves at $8 \mathrm{~h}$ [43]. In Pineapple, AcWRKY35 was induced by drought stress, and 7 AcWRKYs were expressed by cold stress [49]. In Camellia japonica, 13 $C j W R K Y s$ were highly expressed under salicylic acid (SA) stress for $12 \mathrm{~h}$, indicating that the $C j W R K Y$ gene can regulate $C$. japonica to adapt to SA environmental conditions [62].

In the study, we explored the regulatory function of LchiWRKY under abiotic stress by treating somatic embryogenesis of Liriodendron hybrids seedlings with low, high-temperature, and drought stress, and the expression patterns of low temperature, high-temperature, and drought stress in the WRKY family were obtained. Low temperature is one of the major environmental stresses that affect $L$. chinense growth and development, and high temperature and soil drought cause the death of trees and reduce the yield of forests. According to the cold stress expression pattern (Fig. 8), the expression of 11 LchiWRKY genes was significantly induced. The overall expression pattern is divided into two clusters, including high expression at different time points and persistent low expression at all time points. Group I and II responded to cold stress, but group III did not respond, indicating that the response of group III to regulating cold stress was not obvious, while the regulation of groups I and II was more obvious, which also reflected the functional specificity of the group. LchiWRKY5 and 14 may be the main members of the WRKY family in response to low-temperature stress, and $1 \mathrm{~d}$ and $3 \mathrm{~d}$ are the main response time points of the WRKY family. It shows that when $L$. chinense is stimulated by cold stress, it first adapts to the environment through self-protection, and then regulates its adaptability through cold stressrelated resistance genes expressed at $12 \mathrm{~h}$ or $1 \mathrm{~d}$. Group II is the main cold stress regulatory gene, which promotes $L$. chinense to adapt to low-temperature environment stress.

The expression patterns of high-temperature stress were also divided into two groups (Fig. 9), including 1d and 3d high expression patterns and persistent low expression patterns. Similar to cold stress, group III was not significantly induced, and there were more response genes in group II than in group I. LchiWRKY14,18 are the key genes of the WRKY family to respond to hightemperature stress. $1 \mathrm{~d}$ and $3 \mathrm{~d}$ are also the main response time points of high-temperature stress. This also shows that the adaptation of $L$. chinense to a high-temperature environment is similar to that to a low temperature. First, it carries out self-protection and then regulates its adaptability through high-temperature response genes expressed at $12 \mathrm{~h}$ or $1 \mathrm{~d}$. Group II is also the main regulatory gene of $L$. chinense in response to high-temperature stress, which indicates that the function of group II is very important for temperature stress.

The expression patterns of drought stress can also be divided into two groups, containing a high expression pattern of $12 \mathrm{~h}, 1 \mathrm{~d}$, and a persistent low expression pattern (Fig. 10). Different from low temperature and hightemperature stress, group III genes were significantly induced, indicating that group III may be drought-specific response genes. The number of genes in groups I and II are still more than that in group III, indicating that these two groups are also important genes in response to drought stress. LchiWRKY14,18 may be the main response gene and $12 \mathrm{~h}, 1 \mathrm{~d}$ may be the main response time point to drought stress. This shows that $L$. chinense adapts to drought stress as well as temperature stress. It first protects itself and then adapts to the environment by specifically regulating gene expression at $12 \mathrm{~h}$ or $1 \mathrm{~d}$. LchiWRKY family can regulate $L$. chinense to adapt to drought environment, but the regulation function of groups I and II are more obvious than that of group III.

Interestingly, some WRKY genes have high expression patterns under different stresses, such as LchiWRKY5, 23, $14,27,36$, which are highly expressed at $1 \mathrm{~d}$ time points of three stresses, indicating that these genes may have a cross-regulation function under different stresses. These five $L c h i W R K Y s$ were also the key regulatory genes for the growth and development of $L$. chinense to promote $L$. chinense to adapt to different adverse environmental conditions. The results of qRT-PCR under low, hightemperature, and drought stress also showed that $1 \mathrm{~d}$ was the main response time point of the WRKY family, which was consistent with the results of the transcriptome, and reflected the accuracy of transcriptional data.

\section{LchiWRKY transcription factor is mainly expressed in the nucleus}

As transcription factors, they are mainly expressed in the nucleus [40, 49-51, 63], but some are also expressed in other organelles, such as chloroplast, mitochondria, cytoplasm, and cell membrane [43, 51, 63]. Subcellular localization analysis of maize showed that most ZmWRKY transcription factors were expressed in the nucleus, and only a few ZmWRKYs were expressed in 
other organelles [43]. In pineapple, 51 AcWRKYs were located in the nucleus and only 3 in the chloroplast [49]. Similarly, in Orchardgrass, most of $D g W R K Y s$ (88.17\%) were located in the nucleus, and only a few in chloroplasts (7), cytoplasm (2), mitochondria (1), and peroxisome (1) [50]. The difference is that in walnut, all JrWRKYs were expressed in the nucleus [40]. The Subcellular localization analysis of $L$. chinense showed that all LchiWRKYs were expressed in the nucleus, except the LchiWRKY42 was also expressed in the cell membrane. Combined with phylogenetic tree (Fig. 1) analysis, LchiWRKY42 is in a separate branch, so it is speculated that its function is different from other genes, which may be due to functional differentiation in the process of evolution.

The subcellular localization experiments of LchiWRKY18 and LchiWRKY36 in L. chinense showed that these two transcription factors were located in the nucleus (Fig. 14), indicating that they were expressed in the nucleus and regulated the expression of target genes by binding the promoter sequence of target genes. In other species, the subcellular localization experiments of WRKY transcription factor are mainly in the nucleus, For example, in Sugarcane (Saccharum spp.), the subcellular localization experiment of ScWRKY5 showed that it was expressed in the nucleus [64]. In sandalwood (Santalum album L.), the SaWRKY1 protein was also expressed in the nucleus [39]. In Liriodendron hybrids, the LhWOX1 protein was expressed in the nucleus [65].

Based on the above analysis, the expression position of WRKY transcription factor is different in different species, but most members are mainly expressed in the nucleus and a few in other organelles, indicating that the main function of WRKY transcription factors is to regulate gene expression, but also participate in other biological processes.

Overall, the above findings provide insight into the potential functional role of $L$. chinense WRKY genes. The LchiWRKY family has a cross-regulation function, which makes $L$. chinense adapt to different stress environments. Our comprehensive analyses helped select candidate WRKY genes for further functional verification and genetic improvement of L. chinense agronomic traits and environmental resistance. Additionally, to provide a theoretical basis for promoting afforestation of Liriodendron in different areas.

\section{Conclusions}

A comprehensive analysis of the WRKY gene family in $L$. chinense was carried out in the present study. Forty-four full-length WRKY genes were characterized and further classified into three main phylogenetic groups, phylogenetic comparison of WRKY genes from 17 different plant species provided valuable clues about the evolutionary characteristics of LchiWRKY genes, and L. chinense has fewer WRKY members than other higher angiosperms. Lchi WRKY family has a highly similar exon-intron structure and motif compositions within the same subgroup and the regulatory functions of different subgroups are specific. Subcellular localization analysis and experiments showed that LchiWRKYs were mainly expressed in the nucleus, and GO analysis focused on biological processes and molecular functions. The analysis of gene replication events and selection pressure showed that the expansion of the WRKY family was mainly through tandem repeat events, and suffered from purification selection during evolution, which may lead to a small number of WRKY members. More importantly, LchiWRKY is also involved in the regulation of abiotic stress, LchiWRKY5, 23, 14, 27, and 36 cross-regulate the response to low, high-temperature, and drought stress. These results provide a valuable resource for a better understanding of the biological roles of WRKY genes in L. chinense. At the same time, it also provides a theoretical basis for the growth and development and afforestation in different areas of Liriodendron.

\section{Methods}

\section{Datasets and sequence retrieval}

The complete genome, transcript/protein sequences, and genome feature file of $L$. chinense $(L c h i)$ were downloaded from https://www.ncbi.nlm.nih.gov/assembly/GCA 003013855.2. All 16 species contained basal angiosperms Amborella trichopoda, Nymphaea colorata, Magnolia Cinnamomum kanehirae, monocotyledons (Brachypodium distachyon, Panicum hallii, Oryza sativa, Spirodela polyrhiza), dicotyledons (Arabidopsis thaliana, Capsella grandiflora, Cucumis sativus, Eucalyptus grandis, Malus domestica, Gossypium raimondii, Populus trichocarpa, Theobroma cacao, Vitis vinifera), WRKY proteins were obtained from the Phytozome 13 (https://phytozomenext.jgi.doe.gov/), with search in 'WRKY' keyword for these plant species, respectively. Each sequence was checked manually, and the protein sequence without the WRKY domain was deleted.

An HMM (Hidden Markov Model) profile for the WRKY (PFAM ID: PF03106.15) domain (SMART ID: SM00774 and InterPro ID: IPR003657) was retrieved from Pfam (http://pfam.xfam.org) and then used as a query to search all the WRKY domain-containing protein sequences in the $L$. chinense genome using HMMER (v.3.0.1b) with an E-value cut of $<1 \mathrm{E}-5$. Finally, all candidate LchiWRKYs were validated using the Pfam and the Conserved Domains Database (CDD, https://www.ncbi. nlm.nih.gov/Structure/cdd/wrpsb.cgi) to determine that they indeed contained the core domain sequences. 


\section{Sequence analysis}

The gene structure information of each LchiWRKY gene was acquired from the genomic feature file (GFF3) and displayed using GSDS $_{2.0}$ (http://gsds.gao-lab.org/ index.php) [66], while the chromosomal location and microsynteny of LchiWRKYs were visualized using the R package Circlize [67] (https://cran.r-project.org/web/ packages/circlize/). The Multiple Collinearity Scan toolkit (MCScanX) program was used to verify putative paralogous genes (blast hits E-value cutoff $<1 \mathrm{E}-6$, collinearity $>70 \%$ ) [68]. The cis-acting elements of LchiWRKY genes were analyzed by PlantCARE (http://bioin formatics.psb.ugent.be/webtools/plantcare/html/) and displayed using TBtool software [69]. $\mathrm{Ka} / \mathrm{Ks}$ values were

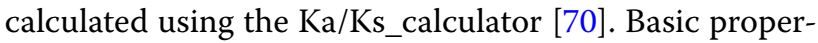
ties of LchiWRKY proteins, including length, molecular weight (MW), and isoelectric point (pI) were calculated using ExPasy (http://au.expasy.org/tools/pi_tool.html) and then subcellular localization was analyzed by PlantmPLoc website (http://www.csbio.sjtu.edu.cn/bioinf/ plant-multi/) [71, 72]. The conserved motifs of LchiWRKY proteins were predicted using MEME(v5.4.1) (https://meme-suite.org/meme/tools/meme) with the following settings: the discovery mode was classic, site distribution was zero or one occurrence (of a contributing motif site) per sequence, the background is 0-order background model, the maximum number of different motifs: 20 , minimum motif width: 6 , and maximum motif width: 50 [35], and displayed using the TBtool software [69]. Gene ontology (GO) analysis was implemented by the clusterProfiler(v4.0.5) R package with LchiWRKY GO id annotated against all GO id of all Lchi genes (Additional file 22: Table S19), in which gene length bias was corrected. GO terms with corrected $P$-value less than 0.05 were considered significantly enriched by LchiWRKY genes [73].

\section{Phylogenetic analysis}

Multiple sequence alignment (MSA) of Amborella trichopoda, Liriodendron chinense, Brachypodium distachyon, Cinnamomum kanehirae, Gossypium raimondii, Oryza sativa, Arabidopsis thaliana, Capsella grandiflora, Cucumis sativus, Eucalyptus grandis, Malus domestica, Nymphaea colorata, Spirodela polyrhiza, Panicum hallii, Populus trichocarpa, Theobroma cacao, Vitis vinifera WRKY domain-containing full-length proteins with FASTA format was done using MUSCLE (v3.8.31) set the following parameters: the maximum number of iterations was 1 with '-maxiters 1', and find diagonals with '-diags -sv -distance1 kbit20_3' [74]. The trimmed MSA file was generated with trimAl (v1.4) [75] set to 'automated1' mode and then used to construct the WRKY phylogenetic tree. The Bayesian phylogenetic tree was constructed using the BEAST software (v2.6.6), by inputting the trimmed file in FASTA format with BEAUti 2 program under the following settings: the Site model was Gamma Site model, substitution rate was 1.0, and substitution model was Dayhoff of site model, clock.rate of clock model was 1.0, Priors of the tree was set to the Yule Model, the Birth Rate was 1.0, the MCMC chain length was $10,000,000$, and then output the file in XML format. After the BEAST program is completed and the TreeAnnotator program set posterior probability limit was 1.0 with a Burnin percentage of 90, target tree type was maximum clade credibility tree, node heights were common ancestor heights to build the Bayesian phylogenetic tree (the Bayesian tree file was shown in Additional files 2 and 3: Tables S2-S3) [76]. The phylogenic tree was then visualized using the $R$ package ggtree [77, 78] (http:// www. bioconductor.org/packages/release/bioc/html/ ggtree.html). OrthoFinder (v2.2.3) was used to explore the WRKY evolutionary status of all the 17 species by using a folder of their protein full-length sequences in the FASTA format with the parameter '-f', and used the DIAMOND sequence search and MSA gene tree inference, then MAFFT was used for multiple sequence alignment and the IQ-TREE program was used to construct species tree with maximum likelihood species tree inference, and the 17 rooted species tree file was shown in (Additional file 4: Table S4) [79-81], and then displayed the rooted species tree by using the $R$ package ggtree [77].

\section{Plant materials and stress treatment}

Liriodendron hybrids seedlings generated through somatic embryogenesis were used as the starting material throughout this study. Before any experiments were performed, plantlets were taken out of the culture medium vessel and acclimatized in a greenhouse for 2 weeks $\left(22^{\circ} \mathrm{C}\right.$, long day photoperiod of $16 \mathrm{~h}$ light $/ 8 \mathrm{~h}$ dark and $75 \%$ relative humidity). For various abiotic stress treatments, plants were transferred to a growth chamber (long time photoperiod of $16 \mathrm{~h}$ light/8h dark and $75 \%$ relative humidity): to simulate cold or heat or drought stress, plantlets were subjected to a $4{ }^{\circ} \mathrm{C}$ or $40^{\circ} \mathrm{C}$ or $15 \%$ PEG6000 (the 15\% concentration was determined according to the reports of references $[47,49,82,83]$ and the preliminary experimental results (Additional file 19: Fig. S3), the control group was added the same amount of water to the substrate and the temperature was $22^{\circ} \mathrm{C}$ ) treatment respectively for $1 \mathrm{~h}, 3 \mathrm{~h}, 6 \mathrm{~h}, 12 \mathrm{~h}, 1 \mathrm{~d}$, and $3 \mathrm{~d}$ in the growth chamber. Each treatment consisted of five biological replicates (five plantlets) for each time point. All treated leaf tissue samples of each plantlet were immediately frozen in liquid nitrogen and stored at $-80^{\circ} \mathrm{C}$ for further RNA extraction. 


\section{RNA extraction, library preparation, and sequencing}

RNA degradation and contamination were monitored on $1 \%$ agarose gels. RNA purity was checked using the NanoPhotometer ${ }^{\circledR}$ spectrophotometer (IMPLEN, CA, USA).RNA integrity was assessed using the RNA Nano 6000 Assay Kit of the Bioanalyzer 2100 system (Agilent Technologies, CA, USA), cDNA was synthesized using a HiScript ${ }^{\circledR}$ III 1st Strand cDNA Synthesis Kit (Vazyme, Nanjing, China) with the extracted RNA as a template.

A total amount of $1 \mu \mathrm{g}$ RNA per sample was used as input material for the RNA sample preparations. Sequencing libraries were generated using NEBNext ${ }^{\circledR}$ UltraTM RNA Library Prep Kit for Illumina ${ }^{\circledR}$ (NEB, USA) following the manufacturer's recommendations and index codes were added to attribute sequences to each sample. Briefly, mRNA was purified from total RNA using poly-T oligo-attached magnetic beads. Fragmentation was carried out using divalent cations under elevated temperature in NEBNext First Strand Synthesis Reaction Buffer (5X). First-strand cDNA was synthesized using random hexamer primer and M-MuLV Reverse Transcriptase (RNase H-). Second strand cDNA synthesis was subsequently performed using DNA Polymerase I and RNase H. Remaining overhangs were converted into blunt ends via exonuclease/polymerase activities. After adenylation of 3'ends of DNA fragments, NEBNext Adaptor with hairpin loop structure was ligated to prepare for hybridization. To select cDNA fragments of preferentially $250 \sim 300 \mathrm{bp}$ in length, the library fragments were purified with AM Pure XP system (Beckman Coulter, Beverly, USA). Then $3 \mu$ l USER Enzyme (NEB, USA) was used with size-selected, adaptor-ligated cDNA at $37^{\circ} \mathrm{C}$ for $15 \mathrm{~min}$ followed by $5 \mathrm{~min}$ at $95^{\circ} \mathrm{C}$ before PCR. Then PCR was performed with Phusion High -Fidelity DNA polymerase, Universal PCR primers, and Index (X) Primer. At last, PCR products were purified (AMPure XP system) and library quality was assessed on the Agilent Bioanalyzer 2100 system.

The clustering of the index-coded samples was performed on a cBot Cluster Generation System using TruSeq PE Cluster Kit v3-cBot-HS (Illumia) according to the manufacturer's instructions. After cluster generation, the library preparations were sequenced on an Illumina Novaseq 6000 platform and $150 \mathrm{bp}$ paired-end reads were generated.

\section{Transcriptome (RNA-seq) analysis}

The L. chinense 7 time points leaf transcriptome with biological triplicates (three plantlets) of heat, cold, and drought stress was analyzed using RNA-seq pipeline, Raw data (raw reads) of FastQ format files were firstly carried out quality control through FastQC (v0.11.9) to obtain the overview of Raw data, including Basic Statistics, Per base sequence quality, Per sequence quality scores, Per sequence GC content, Per base N content, Sequence Length Distribution, and Adapter Content. Then the Raw data were filtered according to the quality control results by using Trimmomatic (v0.39) with the following parameters: remove adapters (ILLUMINACLIP: TruSeq3-PE. fa:2:30:10), remove leading low quality or $\mathrm{N}$ bases (below quality 10) (LEADING:10), remove trailing low quality or $\mathrm{N}$ bases (below quality 10) (TRAILING:10), scan the read with a 4-base wide sliding window, cutting when the average quality per base drops below 20 (SLIDINGWINDOW:4:20), drop reads below the 90 bases long (MINLEN:90) [84]. In this step, clean data (clean reads) were obtained by removing reads containing adapter, reads containing ploy- $\mathrm{N}$ and low-quality reads from Raw data. At the same time, Q20, Q30, and GC content of the clean data were calculated. All the downstream analyses were based on clean data with high quality.

Reference genome and gene model annotation files L. chinense, were downloaded from the NCBI website (https://www.ncbi.nlm.nih.gov/assembly/GCA 003013855.2), index of the reference genome was built using Hisat2 (v2.2.1) and paired-end clean reads were aligned to the reference genome using Hisat2 (v2.2.1) [85]. We selected Hisat2 as the mapping tool for that Hisat2 can generate a database of splice junctions based on the gene model annotation file and thus a better mapping result than other non-splice mapping tools. Then we used the Kallisto (v0.46.1) to quantify gene expression by calculating all clean reads mapped to this gene with "kallisto quant" command, and count Transcripts Per Million (TPM) values of individual genes, considering the effect of sequencing depth and gene length for the reads count at the same time and is currently the most commonly used method for estimating gene expression levels [86]. We used DESeq2 (v1.16.1) with the (ANOVA method: p.adjust<0.05, $|\log 2 \mathrm{FC}|>1)$ to determine differentially expressed genes (DEGs), and TPM values were used to calculate the transcript abundance of LchiWRKYs. All $L$. chinense genes had been annotated previously in the Pfam database (http://pfam.xfam.org/) and Blast2GO website (http://www.blast2go.com). The data quality, mapping rate, gene annotation, and normalization (TPM values) of heat, cold, and drought stress RNAseq are shown in (Additional files 20,21, 22, and 23: Tables S17-S20). Expression heatmaps were created using the pheatmap (v1.0.12) R package, based on the transformed data of $\log 2(\mathrm{TPM}+1)$ values. The transcriptome data used in this study has been archived and can also be obtained on the NCBI website, cold and heat stress accession numbers were PRJNA679089 (https://www.ncbi.nlm.nih.gov/bioproject/PRJNA 
679089/), and drought stress accession number was PRJNA679101 (https://www.ncbi.nlm.nih.gov/biopr oject/PRJNA679101/).

\section{qRT-PCR analysis}

Primers used for quantifying the expression of LchiWRKYs were designed by using the primer3 website (https://www.yeastgenome.org/primer3) (Additional file 24: Table S21). qRT-PCR was carried out with a Roche Lightcyler ${ }^{\circledR} 480$ II instrument using 2x Ace ${ }^{\circledR}$ qPCR SYBR ${ }^{\circledR}$ Green Master Mix (Without ROX) (Vazyme, Nanjing, China). The composition of the PCR mix was as follows: $10 \mu \mathrm{l} 2 \mathrm{x}$ AceQ ${ }^{\circledR}$ qPCR $\mathrm{SYBR}^{\circledR}$ Green Master Mix (Without ROX), 0.4 $\mu$ l of each primer and $1 \mu \mathrm{l}$ of cDNA template $(10 \mathrm{ng} / \mu \mathrm{l})$, add-

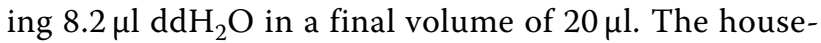
keeping $L$. chinense $18 S$ gene was used as an internal control. The melt and standard curve of WRKY qRTPCR primers were shown in Additional file 25: Fig. S4. The reaction was carried out as follows: $95^{\circ} \mathrm{C}$ for $10 \mathrm{~min}$, followed by 45 cycles of $95^{\circ} \mathrm{C}$ for $10 \mathrm{~s}$, then $60^{\circ} \mathrm{C}$ for $30 \mathrm{~s}$. All reactions were run in 96-well plates. Each reaction was performed in biological triplicates, as well as three technical replicates. All data generated from real-time PCR amplification was analyzed using a $2-\triangle \triangle \mathrm{CT}$ method.

\section{Subcellular localization experiment of LchiWRKY18 and LChiWRKY36}

The RNA extracted from the leaves of Liriodendron hybrids was reversed into cDNA, and the full-length sequences of LchiWRKY18 and 36 CDS were cloned. The LchiWRKY18 and 36 sequences were recombined by digesting the HindIII and XbaI sites of empty vector pJIT166-GFP to obtain pJIT166-LchiWRKY18-GFP and pJIT166-LchiWRKY36-GFP fusion expression vectors. The primers of the LchiWRKY18 recombination vector were 5 '-atttggagaggacagcccaagcttCATGCTTAGAATGGAGGACTCAC-3' (forward direction) and $5^{\prime}$-ccttgctcaccatggatcctctagaCAA GAGGGAAGAACAAGGAT- $3^{\prime}$ (the reverse direction). The primers of the LchiWRKY36 recombination vector were $5^{\prime}$-atttggagaggacagcccaagcttCAT GGAGCAGTTGATCTTTATGTT-3' (forward direction) and $5^{\prime}$-ccttgctcaccatggatcctctagaCAAGGGTCG AACACGAG-3'(the reverse direction). The vector map of pJIT166-GFP,pJIT166-LchiWRKY18-GFP, and pJIT166-LchiWRKY36-GFP were in Additional file 26: Fig. S5.

The callus of Liriodendron hybrids cultured for 20 days was used to prepare protoplasts, and protoplasts were slowly and gently dissolved into a solution $(10 \mathrm{~mL})$ containing $0.5 \mathrm{Mmannitol}, 20 \mathrm{mM}$ MES, pH 5.7, $20 \mathrm{mM} \mathrm{KCl,} \mathrm{0.1 \%} \mathrm{(w/v)} \mathrm{bovine} \mathrm{serum}$ albumin, $10 \mathrm{mM} \mathrm{CaCl}$. and digested at $28^{\circ} \mathrm{C}$ under dark conditions for $3 \mathrm{~h}$ [65], The protoplasts were transformed by PEG-6000, pipetted into a 6-well cell culture plate, and cultured at $23^{\circ} \mathrm{C}$ under dark for $16 \sim 48 \mathrm{~h}$ [65], then the fluorescence effect of protoplasts was observed by ZEISS LSM 800 fluorescence microscope (Carl Zeiss, Germany).

\section{Abbreviations \\ Lchi: Liriodendron chinense; WD: WRKY Domain; AtWRKY: A. thaliana WRKY; AmtrWRKY: A. trichopoda WRKY; BdWRKY: B. distachyon WRKY; CgWRKY: C. grandiffora WRKY; CsWRKY: C. sativus WRKY; CKWRKY: C. kanehirae WRKY; EgWRKY: E. grandis WRKY; GraWRKY: G. raimondii WRKY; LchiWRKY: L. chinense WRKY: MdWRKY: M. domestica WRKY; NcWRKY: N. colorata WRKY; OsWRKY: O. sativa WRKY; PhWRKY: P. hallii WRKY; PtrWRKY: P. trichocarpa WRKY; SpWRKY: S. polyrhiza WRKY; TcWRKY: T. cacao WRKY; VVWRKY: V. vinifera WRKY; TFs: \\ Transcription factors; WGD: Whole-genome duplication; qRT-PCR: Quantita- tive real-time PCR; GO: Gene ontology; MW: Protein molecular weight; pl: Isoelectric point; aa: Amino acids; MSA: Multiple sequence alignment; DEGs: Differentially Expressed Genes; TPM: Transcripts Per Million; SA: Salicylic Acid; ABA: Abscisic Acid; HMM: Hidden Markov Model; FPKM: Fragments Per Kilo- base of exon model per Million mapped fragments; PIN: PIN-FORMED; GAox: Gibberellin oxidase; CBF: C-regeneration binding factors; Al: Aluminum; GAD: Glutamate decarboxylase; MYB: Myeloblastosis.}

\section{Supplementary Information}

The online version contains supplementary material available at https://doi. org/10.1186/s12870-021-03371-1.

Additional file 1: Table S1. Physicochemical properties and chromosomal details of LchiWRKY proteins.

Additional file 2: Table S2. LChiWRKYs Bayesian Phylogenetic tree

Additional file 3: Table S3. 1308 WRKY protein sequences and Bayesian phylogenetic tree of the 17 species.

Additional file 4: Table S4. The Gene ID and corresponding subgroup information and rooted species tree of WRKY family of 17 species.

Additional file 5: Table S5. Chromosome length and location of LChiWRKYS.

Additional file 6: Table S6. List of tandem replicated LchiWRKYS. Additional file 7: Table S7. Motifs identified in the LchiWRKYS. Additional file 8: Figure S1. GO enrichment analysis of LchiWRKY genes. Additional file 9: Table S8. Number and length of LChiWRKY exons and introns.

Additional file 10: Figure S2. Multiple sequence alignment of LchiWRKYS.

Additional file 11: Table S9. Domain sequence and groups of LchiWRKYS. Additional file 12: Table S10. List of cis-regulatory elements (CREs) present in the promoter region of identified LChiWRKYS.

Additional file 13: Table S11. LchiWRKY's TPM value of cold stress Additional file 14: Table S12. LchiWRKY's TPM value of heat stress Additional file 15: Table S13. LchiWRKY's TPM value of drought stress. Additional file 16: Table S14. Standardization data of GRT-PCR conditions for cold stress. 
Additional file 17: Table S15. Standardization data of qRT-PCR conditions for heat stress.

Additional file 18: Table S16. Standardization data of aRT-PCR conditions for drought stress.

Additional file 19: Figure S3. 15\% PEG6000 pre-experimental phenotype.

Additional file 20: Table S17. RNA-seq quality data for three stress experiments.

Additional file 21: Table S18. Mapping rate of reference genome used for RNA-seq of three stress experiments.

Additional file 22: Table S19. Genome-wide gene Pfam and GO annotation of Lchi.

Additional file 23: Table S20. All DEGs TPM values from three stress experiments.

Additional file 24: Table S21. List of primers used in the GRT-PCR analysis for the selected LchiWRKYS.

Additional file 25: Figure S4. Melt and standard curve of qRT-PCR primers of LChiWRKYs.

Additional file 26: Figure S5. Three fusion expression vector Maps.

\section{Acknowledgments}

Not applicable.

\section{Authors' contributions}

WHW performed the genome-wide analysis of LChiWRKYS, qPCR, and subcelIular localization experiments and wrote the manuscript. S Z assisted with data analysis. LX assisted subcellular localization experiments, LMZ, DDW, SQL, YLiu, YLu performed the promoter design and qPCR experiments. ZDH, LMY, JHC, JSS were responsible for the overall concept and experimental designs, data integration, analysis and interpretation, and manuscript preparation. All authors approved the final manuscript.

\section{Funding}

This work was supported by The National Natural Science Foundation of China (32071784), the Key Research and Development Plan of Jiangsu Province (BE2017376), the Qinglan Project of Jiangsu Province, Distinguished Professor Project of Jiangsu province and the Priority Academic Program Development of Jiangsu Higher Education Institutions (PAPD). The funding bodies had no role in the design of the study and collection, analysis and interpretation of data or in writing the manuscript.

\section{Availability of data and materials}

All data analyzed during this study are included in this article and its additional files.

\section{Declarations}

\section{Ethics approval and consent to participate}

Not applicable.

\section{Consent for publication}

Not applicable.

\section{Competing interests}

The authors declare that they have no competing interests.

\section{Author details}

${ }^{1}$ Key Laboratory of Forest Genetics and Biotechnology, Ministry of Education of China, Co-Innovation Center for the Sustainable Forestry in Southern China, Nanjing Forestry University, Nanjing, China. ${ }^{2}$ College of Biology and the Environment, Nanjing Forestry University, Nanjing, China.

Received: 14 September 2021 Accepted: 29 November 2021 Published online: 10 January 2022

\section{References}

1. Chen F, Hu Y, Vannozzi A, Wu K, Cai H, Qin Y, et al. The WRKY transcription factor family in model plants and crops. Crit Rev Plant Sci. 2018;36(5-6):311-35.

2. Ryu H, Han M, Lee S, Cho J, Ryoo N, Heu S, et al. A comprehensive expression analysis of the WRKY gene superfamily in rice plants during defense response. Plant Cell Rep. 2006;25(8):836-47.

3. Rinerson Cl, Rabara RC, Tripathi P, Shen QJ, Rushton PJ. The evolution of WRKY transcription factors. BMC Plant Biol. 2015;15(1):66.

4. Chen C, Chen X, Han J, Lu W, Ren Z. Genome-wide analysis of the WRKY gene family in the cucumber genome and transcriptome-wide identification of WRKY transcription factors that respond to biotic and abiotic stresses. BMC Plant Biol. 2020;20(1):1-9.

5. Wan Y, Mao M, Wan D, Yang Q, Yang F, Mandlaa, et al. Identification of the WRKY gene family and functional analysis of two genes in Caragana intermedia. BMC Plant Biol. 2018;18(1):31.

6. Rushton PJ, Somssich IE, Ringler P, Shen QJ. WRKY transcription factors. Trends Plant Sci. 2010;15(5):247-58.

7. Zhou H, Li Y, Zhang Q, Ren S, Shen Y, Qin L, et al. Genome-wide analysis of the expression of WRKY family genes in different developmental stages of wild strawberry (Fragaria vesca) fruit. PLoS One. 2016;11(5):e154312.

8. Banerjee A, Roychoudhury A. WRKY proteins: signaling and regulation of expression during abiotic stress responses. Sci World J. 2015;2015:1-17.

9. Ling J, Jiang W, Zhang Y, Yu H, Mao Z, Gu X, et al. Genome-wide analysis of WRKY gene family in Cucumis sativus. BMC Genomics. 2011;12(1):471.

10. Phukan UJ, Jeena GS, Shukla RK. WRKY transcription factors: molecular regulation and stress responses in plants. Front Plant Sci. 2016;7:760.

11. Song $H$, Sun W, Yang G, Sun J. WRKY transcription factors in legumes. BMC Plant Biol. 2018;18(1):243.

12. Zhang $Y$, Wang $L$. The WRKY transcription factor superfamily: its origin in eukaryotes and expansion in plants. BMC Evol Biol. 2005;5(1):1.

13. Wu K, Guo Z, Wang H, Li J. The WRKY family of transcription factors in rice and Arabidopsis and their origins. DNA Res. 2005;12(1):9-26.

14. Yue $H$, Chang X, Zhi Y, Wang L, Xing G, Song W, et al. Evolution and identification of the WRKY gene family in quinoa (Chenopodium quinoa). Genes-Basel. 2019;10(2):131.

15. Wang Y, Feng L, Zhu Y, Li Y, Yan H, Xiang Y. Comparative genomic analysis of the WRKY III gene family in populus, grape, arabidopsis and rice. Biol Direct. 2015;10(1):1-27.

16. Li Y, Zhang L, Zhu P, Cao Q, Sun J, Li Z, et al. Genome-wide identification, characterisation and functional evaluation of WRKY genes in the sweet potato wild ancestor Ipomoea trifida (H.B.K.) G. Don. under abiotic stresses. BMC Genet. 2019;20(1):1-5.

17. Song Y, Cui H, Shi Y, Xue J, Ji C, Zhang C, et al. Genome-wide identification and functional characterization of the Camelina sativa WRKY gene family in response to abiotic stress. BMC Genomics. 2020;21(1):1-7.

18. Guo C, Guo R, Xu X, Gao M, Li X, Song J, et al. Evolution and expression analysis of the grape (Vitis vinifera L.) WRKY gene family. J Exp Bot. 2014;65(6):1513-28.

19. Ye H, Qiao L, Guo H, Guo L, Ren F, Bai J, et al. Genome-wide identification of wheat WRKY gene family reveals that TaWRKY75-A is referred to drought and salt resistances. Front Plant Sci. 2021;12:812.

20. Wei L, Hong W, Diqiu Y. Arabidopsis WRKY transcription factors WRKY12 and WRKY13 oppositely regulate flowering under short-day conditions. Mol Plant. 2016;9(11):1492-503.

21. Niu C, Wei W, Zhou Q, Tian A, Hao Y, Zhang W, et al. Wheat WRKY genes TaWRKY2 and TaWRKY19 regulate abiotic stress tolerance in transgenic Arabidopsis plants. Plant Cell Environ. 2012;35(6):1156-70.

22. Aihong $Y$, Christopher WD, Xiaohong $Y$, Hongwen $\mathrm{H}$. Impacts of biogeographic history and marginal population genetics on species range limits: a case study of Liriodendron chinense. Sci Rep-UK. 2016;6(1):1-2.

23. Chen J, Hao Z, Guang X, Zhao C, Wang P, Xue L, et al. Liriodendron genome sheds light on angiosperm phylogeny and species-pair differentiation. Nat Plants. 2019:5(1):18-25.

24. Sheng Y, Hao Z, Peng Y, Liu S, Hu L, Shen Y, et al. Morphological, phenological, and transcriptional analyses provide insight into the diverse flowering traits of a mutant of the relic woody plant Liriodendron chinense. Hortic Res-England. 2021;8(1):1-6.

25. Hu L, Wang P, Long X, Wu W, Zhang J, Pan Y, et al. The PIN gene family in relic plant L. chinense: genome-wide identification and gene expression 
profiling in different organizations and abiotic stress responses. Plant Physiol Biochem. 2021;162:634-46.

26. Hu L, Wang P, Hao Z, Lu Y, Xue G, Cao Z, et al. Gibberellin oxidase gene family in L. chinense: genome-wide identification and gene expression analysis. Int J Mol Sci. 2021;22(13):7167.

27. Wu W, Zhu S, Zhu L, Wang D, Liu Y, Liu S, et al. Characterization of the Liriodendron Chinense MYB gene family and its role in abiotic stress response. Front Plant Sci. 2021;12:641280.

28. Guan Y, Liu S, Wu W, Hong K, Li R, Zhu L, et al. Genome-wide identification and cold stress-induced expression analysis of the CBF gene family in Liriodendron chinense. J Forestry Res. 2021;32:2531-43.

29. Zhong YD, Sun XY, Liu EY, Li YQ, Gao Z, Yu FX. Expressed sequence tag analysis of functional genes associated with adventitious rooting in Liriodendron hybrids. Genet Mol Res. 2016;15(2):10.4238.

30. LiT, Yuan W, Qiu S, Shi J. Selection of reference genes for gene expression analysis in Liriodendron hybrids' somatic embryogenesis and germinative tissues. Sci Rep. 2021;11(1):4957.

31. Wang P, Dong Y, Zhu L, Hao Z, Hu L, Hu X, et al. The role of Y-aminobutyric acid in aluminum stress tolerance in a woody plant, Liriodendron chinense $\times$ tulipifera. Hortic Res-England. 2021;8(1):1-5.

32. Chaw S, Liu Y, Wu Y, Wang H, Lin Cl, Wu C, et al. Stout camphor tree genome fills gaps in understanding of flowering plant genome evolution. Nat Plants. 2019;5(1):63-73.

33. Ross CA, Liu Y, Shen QJ. The WRKY gene family in rice (Oryza sativa). J Integr Plant Biol. 2007;49(6):827-42.

34. Hurst LD. The Ka/Ks ratio: diagnosing the form of sequence evolution. Trends Genet. 2002;18(9):486.

35. Bailey TL, Johnson J, Grant CE, Noble WS. The MEME suite. Nucleic Acids Res. 2015:43(W1):W39-49.

36. Abdullah, Faraji S, Mehmood F, Hafiz MTM, Ahmed I, Heidari P, et al. The GASA gene family in cacao (Theobroma cacao, Malvaceae): genome wide identification and expression analysis. Agronomy (Basel). 2021;11(1425):1425.

37. Musavizadeh Z, Najafi-Zarrini H, Kazemitabar SK, Hashemi SH, Faraji S, Barcaccia $G$, et al. Genome-wide analysis of potassium channel genes in rice: expression of the OsAKT and OsKAT genes under salt stress. Genes-Basel. 2021;12(5):784.

38. Tiika RJ, Wei J, Ma R, Yang H, Cui G, Duan H, et al. Identification and expression analysis of the WRKY gene family during different developmental stages in Lycium ruthenicum Murr. fruit. PeerJ. 2020;8:e10207.

39. Yan H, Li M, Xiong Y, Wu J, Da Silva JAT, Ma G. Genome-wide characterization, expression profile analysis of WRKY family genes in Santalum album and functional identification of their role in abiotic stress. Int J Mol Sci. 2019;20(22):5676.

40. Hao F, Yang G, Zhou H, Yao J, Liu D, Zhao P, et al. Genome-wide identification and transcriptional expression profiles of transcription factor WRKY in common Walnut (Juglans regia L.). Genes-Basel. 2021;12(9):1444.

41. Sun W, Ma Z, Chen H, Liu M. Genome-wide investigation of WRKY transcription factors in Tartary buckwheat (Fagopyrum tataricum) and their potential roles in regulating growth and development. PeerJ. 2020;8:e8727.

42. Li D, Liu P, Yu J, Wang L, Dossa K, Zhang Y, et al. Genome-wide analysis of WRKY gene family in the sesame genome and identification of the WRKY genes involved in responses to abiotic stresses. BMC Plant Biol. 2017;17(1):152.

43. Hu W, Ren Q, Chen Y, Xu G, Qian Y. Genome-wide identification and analysis of WRKY gene family in maize provide insights into regulatory network in response to abiotic stresses. BMC Plant Biol. 2021;21(1):427.

44. Nan H, Lin Y, Liu J, Huang H, Li W, Gao L. Genome-wide analysis of the WRKY transcription factor gene family and their response to salt stress in rubber tree. Trop Plant Biol. 2021;14(1):22-33.

45. Yuan H, Guo W, Zhao L, Yu Y, Chen S, Tao L, et al. Genome-wide identification and expression analysis of the WRKY transcription factor family in flax (Linum usitatissimum L.). BMC Genomics. 2021;22(1):375.

46. Zheng J, Zhang Z, Tong T, Fang Y, Zhang X, Niu C, et al. Genome-wide identification of WRKY gene family and expression analysis under abiotic stress in barley. Agronomy. 2021;11(3):521.
47. Qu R, Cao Y, Tang X, Sun L, Wei L, Wang K. Identification and expression analysis of the WRKY gene family in Isatis indigotica. Gene. 2021;783:145561.

48. Chen Y, Jing X, Wang S, Wang J, Zhang S, Shi Q. Genome-wide analysis of WRKY transcription factor family in melon (Cucumis Melo L.) and their response to powdery mildew. Plant Mol Biol Rep. 2021;1-14. https://doi. org/10.1007/s11105-020-01271-6.

49. Xie T, Chen C, Li C, Liu J, Liu C, He Y. Genome-wide investigation of WRKY gene family in pineapple: evolution and expression profiles during development and stress. BMC Genomics. 2018;19(1):1-8.

50. Ren J, Hu J, Zhang A, Ren S, Jing T, Wang X, et al. The whole-genome and expression profile analysis of WRKY and RGAs in Dactylis glomerata showed thatDG6C02319.1 and DgWRKYs may cooperate in the immunity against rust. PeerJ. 2021;9:e11919.

51. Jeyasri R, Muthuramalingam P, Satish L, Adarshan S, Lakshmi MA, Pandian SK, et al. The role of OsWRKY genes in rice when faced with single and multiple abiotic stresses. Agronomy. 2021;11(7):1301.

52. Li L, Liu Q, Liu T, Cui X, Ning W. Expression of putative luteolin biosynthesis genes and WRKY transcription factors in Taraxacum antungense kitag. Plant Cell Tissue Organ Cult. 2021;145(3):649-65.

53. Wang N, Xia E, Gao L. Genome-wide analysis of WRKY family of transcription factors in common bean, Phaseolus vulgaris: chromosomal localization, structure, evolution and expression divergence. Plant Gene. 2016;5:22-30.

54. Song H, Wang P, Lin J, Zhao C, Bi Y, Wang X. Genome-wide identification and characterization of WRKY gene family in peanut. Front Plant Sci. 2016;7:534.

55. Xie Z, Zhang Z, Zou X, Huang J, Ruas P, Thompson D, et al. Annotations and functional analyses of the rice WRKY gene superfamily reveal positive and negative regulators of abscisic acid signaling in Aleurone cells. Plant Physiol. 2005;137(1):176-89.

56. Zhang L, Chen F, Zhang X, Li Z, Zhao Y, Lohaus R, et al. The water lily genome and the early evolution of flowering plants. Nature. 2020;577(7788):79-84.

57. Wang $Q$, Wang M, Zhang X, Hao B, Kaushik SK, Pan Y. WRKY gene family evolution in Arabidopsis thaliana. Genetica. 2011;139(8):973-83.

58. Jiang Y, Guo L, Ma X, Zhao X, Jiao B, Li C, et al. The WRKY transcription factors PtrWRKY18 and PtrWRKY35 promote Melampsora resistance in Populus. Tree Physiol. 2017;37(5):665-75.

59. Waqas M, Azhar MT, Rana IA, Azeem F, Ali MA, Nawaz MA, et al. Genomewide identification and expression analyses of WRKY transcription factor family members from chickpea (Cicer arietinum L.) reveal their role in abiotic stress-responses. Genes Genomics. 2019;41(4):467-81.

60. Huang K, Wu T, Ma Z, Li Z, Chen H, Zhang M, et al. Rice transcription factor OsWRKY55 is involved in the drought response and regulation of plant growth. Int J Mol Sci. 2021;22(9):4337.

61. Javadi SM, Shobbar ZS, Ebrahimi A, Shahbazi M. New insights on key genes involved in drought stress response of barley: gene networks reconstruction, hub, and promoter analysis. J Genet Eng Biotechnol. 2021;19(1):2.

62. Yang X, Zhou Z, Fu M, Han M, Liu Z, Zhu C, et al. Transcriptome-wide identification of WRKY family genes and their expression profiling toward salicylic acid in Camellia japonica. Plant Signal Behav. 2021;16(1):1844508.

63. Chen Z, Shen Z, Zhao D, Xu L, Zhang L, Zou Q. Genome-wide analysis of LysM-containing gene family in wheat: structural and phylogenetic analysis during development and defense. Genes-Basel. 2021;12(1):31.

64. Wang D, Wang L, Su W, Ren Y, You C, Zhang C, et al. A class III WRKY transcription factor in sugarcane was involved in biotic and abiotic stress responses. Sci Rep-UK. 2020;10(1):1-5.

65. Huo A, Chen Z, Wang P, Yang L, Wang G, Wang D, et al. Establishment of transient gene expression systems in protoplasts from Liriodendron hybrid mesophyll cells. PLoS One. 2017;12(3):e172475.

66. Hu B, Jin J, Guo AY, Zhang H, Luo J, Gao G. GSDS 2.0: an upgraded gene feature visualization server. Bioinformatics. 2015:31(8):1296-7.

67. Gu Z, Gu L, Eils R, Schlesner M, Brors B. circlize implements and enhances circular visualization in R. Bioinformatics. 2014;30(19):2811-2.

68. Wang Y, Tang H, DeBarry JD, Tan X, Li J, Wang X, et al. MCScanX: a toolkit for detection and evolutionary analysis of gene synteny and collinearity. Nucleic Acids Res. 2012;40(7):e49. 
69. Chen C, Chen H, Zhang Y, Thomas HR, Frank MH, He Y, et al. TBtools: an integrative toolkit developed for interactive analyses of big biological data. Mol Plant. 2020;13(8):1194-202.

70. Zhang Z, Li J, Zhao XQ, Wang J, Wong GK, Yu J. KaKs_Calculator: calculating Ka and Ks through model selection and model averaging. Genomics Proteomics Bioinformatics. 2006;4(4):259-63.

71. Chou KC, Shen HB. Plant-mPLoc: a top-down strategy to augment the power for predicting plant protein subcellular localization. PLoS One. 2010;5(6):e11335.

72. Chou K, Shen H. Large-scale plant protein subcellular location prediction. J Cell Biochem. 2007;100(3):665-78.

73. Yu G, Wang L, Han Y, He Q. clusterProfiler: an R package for comparing biological themes among gene clusters. OMICS. 2012;16(5):284-7.

74. Edgar RC. MUSCLE: multiple sequence alignment with high accuracy and high throughput. Nucleic Acids Res. 2004;32(5):1792-7.

75. Capella-Gutierrez S, Silla-Martinez JM, Gabaldon T. trimAl: a tool for automated alignment trimming in large-scale phylogenetic analyses. Bioinformatics. 2009;25(15):1972-3.

76. Bouckaert R, Vaughan TG, Barido-Sottani J, Duchêne S, Fourment M, Gavryushkina A, et al. BEAST 2.5: an advanced software platform for Bayesian evolutionary analysis. PLoS Comput Biol. 2019;15(4):e1006650.

77. Yu G. Using ggtree to visualize data on tree-like structures. Curr Protoc Bioinformatics. 2020;69(1):e96.

78. Yu G, Lam TT, Zhu H, Guan Y. Two methods for mapping and visualizing associated data on phylogeny using Ggtree. Mol Biol Evol. 2018;35(12):3041-3.

79. Emms DM, Kelly S. OrthoFinder: solving fundamental biases in whole genome comparisons dramatically improves orthogroup inference accuracy. Genome Biol. 2015;16(1):1-4.

80. Emms DM, Kelly S. OrthoFinder: phylogenetic orthology inference for comparative genomics. Genome Biol. 2019;20(1):1-4

81. Emms DM, Kelly S. STRIDE: species tree root inference from gene duplication events. Mol Biol Evol. 2017;34(12):3267-78.

82. Cheng Y, Ahammed GJ, Yao Z, Ye Q, Ruan M, Wang R, et al. Comparative genomic analysis reveals extensive genetic variations of WRKYs in Solanaceae and functional variations of CaWRKYs in pepper. Front Genet. 2019;10:492.

83. Muthamilarasan M, Bonthala VS, Khandelwal R, Jaishankar J, Shweta S Nawaz K, et al. Global analysis of WRKY transcription factor superfamily in Setaria identifies potential candidates involved in abiotic stress signaling. Front Plant Sci. 2015;6:910

84. Bolger AM, Lohse M, Usadel B. Trimmomatic: a flexible trimmer for Illumina sequence data. Bioinformatics. 2014;30(15):2114-20.

85. Kim D, Paggi JM, Park C, Bennett C, Salzberg SL. Graph-based genome alignment and genotyping with HISAT2 and HISAT-genotype. Nat Biotechnol. 2019;37(8):907-15.

86. Bray NL, Pimentel H, Melsted P, Pachter L. Near-optimal probabilistic RNAseq quantification. Nat Biotechnol. 2016;34(5):525-7.

\section{Publisher's Note}

Springer Nature remains neutral with regard to jurisdictional claims in published maps and institutional affiliations.

Ready to submit your research? Choose BMC and benefit from:

- fast, convenient online submission

- thorough peer review by experienced researchers in your field

- rapid publication on acceptance

- support for research data, including large and complex data types

- gold Open Access which fosters wider collaboration and increased citations

- maximum visibility for your research: over $100 \mathrm{M}$ website views per year

At $\mathrm{BMC}$, research is always in progress.

Learn more biomedcentral.com/submissions 